\title{
Average Complexity of a Gift-Wrapping Algorithm for Determining the Convex Hull of Randomly Given Points
}

\author{
K. H. Borgwardt
}

Institut fuer Mathematik, Universitaet Augsburg, Universitaetsstrasse 14, D-86135 Augsburg, Germany

borgwardt@uni-augsburg.de

\begin{abstract}
This paper presents an algorithm and its probabilistic analysis for constructing the convex hull of $m$ given points in $\mathbb{R}^{n}$, the $n$-dimensional Euclidean space. The algorithm under consideration combines the Gift-Wrapping concept with the so-called Throw-Away Principle (introduced by Akl and Toussaint [1] and later by Devroye [10]) for nonextremal points. The latter principle had been used for a convex-hull-construction algorithm in $\mathbb{R}^{2}$ and for its probabilistic analysis in a recent paper by Borgwardt et al. [5]. There, the considerations remained much simpler, because in $\mathbb{R}^{2}$ the construction of the convex hull essentially requires recognition of the extremal points and of their order only.

In this paper the Simplex method is used to organize a walk over the surface of the convex hull. During this walk all facets are discovered. Under the condition of general position this information is sufficient, because the whole face lattice can simply be deduced when the set of facets is available.

Exploiting the advantages of the revised Simplex method reduces the update effort to an $n \times n$ matrix and the number of calculated quotients for the pivot search to the points which are not thrown away.

For this algorithm a probabilistic analysis can be carried out. We assume that our $m$ random points are distributed identically, independently, and symmetrically under rotations in $\mathbb{R}^{n}$. Then the calculation of the expected effort becomes possible for a whole parametrical class of distributions over the unit ball. The results mean a progress in three directions:

- a parametrization of the expected effort can be given;

- the dependency on $n$-the dimension of the space-can be evaluated;

- the additional work of preprocessing for detecting the vertices can be avoided without losing its advantages.
\end{abstract}




\section{Introduction}

The Problem and Its Geometry

Let $m$ points $a_{1}, \ldots, a_{m} \in \mathbb{R}^{n}$ be given. We want to construct or determine the convex hull of these points, which is denoted by $Y:=C H\left(a_{1}, \ldots, a_{m}\right)$. In general, we base our considerations on the following:

\section{Condition of Nondegeneracy.}

Each subset of $n$ vectors out of $\left\{a_{1}, \ldots, a_{m}\right\}$ is linearly independent and each subset of $n+1$ such points is in general position.

It is our aim to study the expected number of arithmetical operations for determining the convex hull under several specified stochastic models, i.e., assumptions on the distribution of the data $a_{1}, \ldots, a_{m}$. Here we concentrate on distributions where our condition of nondegeneracy is satisfied almost surely, i.e., with probability 1 . Since the computational effort is bounded for the treatment of $m$ points, ignoring the degeneracy cases completely for the purpose of calculating the expected values is permitted.

In addition, our condition of nondegeneracy makes sure that the polyhedron $Y$ becomes simplicial. Hence, all facets of $Y$ are $(n-1)$-dimensional boundary simplices. All the other (lower-dimensional) faces of $Y$ can simply be obtained by dropping some of the points generating a certain facet. For instance, let $a_{1}, \ldots, a_{n}$ generate a facet, i.e., $C H\left(a_{1}, \ldots, a_{n}\right)$ is a facet. Then $C H\left(a_{1}, \ldots, a_{k}\right)$ (with $\left.1 \leqq k \leqq n\right)$ is a $(k-1)$ dimensional face of $Y$ and every face can be obtained in that way. Now it is clear that knowing the facet set is sufficient.

Hence, we are allowed to formulate a "reduced task":

Determine all facets of $Y$.

We denote the set of $Y$-facets by $\mathfrak{F}$.

The author has studied such polytopes $C H\left(a_{1}, \ldots, a_{m}\right)$ thoroughly in [4] for the purpose of calculating the number of iteration steps required by the Simplex Algorithm, both from a geometrical and from a probabilistic point of view. There the Simplex Algorithm moved on a path over successively adjacent facets from a start facet to a target or final facet. This time, we move in the same way, but with the aim of visiting every facet of $Y$.

Before discussing some popular concepts for constructing convex hulls (including our own), we summarize some important geometrical properties of the surface of $Y$.

Definition 1.1. Let $\Delta$ be an $n$-tupel $\left(\Delta^{1}, \ldots, \Delta^{n}\right)$ with components $\Delta^{i} \in\{1, \ldots, m\}$ for $i=1, \ldots, n$ and $\Delta^{i}<\Delta^{i+1}$ for $i=1, \ldots, n-1$. Further, let $\mathfrak{F}$ be the set of facets of $Y$, which is the set of all $(n-1)$-dimensional faces. Accordingly, $\mathcal{V}$ denotes the set of all vertices or extremal points of $Y$. It is intuitively clear that $\mathcal{V} \subset\left\{a_{1}, \ldots, a_{m}\right\}$. By $\# \mathfrak{F}$ and $\# \mathcal{V}$ we denote the cardinalities (number of elements) of the corresponding sets.

From pp. 62-95 of [4] the following facts are known. 


\section{Lemma 1.1.}

(a) Every facet is a boundary simplex of the type $C H\left(a_{\Delta^{\prime}}, \ldots, a_{\Delta^{\prime \prime}}\right)$, which is generated by $n$ points $a_{\Delta^{1}}, \ldots, a_{\Delta^{n}}$.

(b) Every boundary simplex itself is bounded by its $n$ different $(n-2)$-dimensional faces, which we call the side of a facet or the border of a boundary simplex or simply ridge. That means that a facet $\mathrm{CH}\left(a_{\Delta^{\prime}}, \ldots, a_{\Delta^{n}}\right)$ possesses the $n$ borders (ridges) $C H\left(a_{\Delta^{1}}, \ldots, a_{\Delta^{n-1}}\right), \ldots, C H\left(a_{\Delta^{1}}, \ldots, a_{\Delta^{i-1}}, a_{\Delta^{i+1}}, \ldots, a_{\Delta^{n}}\right), \ldots$, $\mathrm{CH}\left(a_{\Delta^{2}}, \ldots, a_{\Delta^{n}}\right)$.

(c) To each border of a boundary simplex (ridge) exactly two boundary simplices or facets are incident. That means:

If $\mathrm{CH}\left(a_{\Delta^{1}}, \ldots, a_{\Delta^{i-1}}, a_{\Delta^{i+1}}, \ldots, a_{\Delta^{n}}\right)$ is a ridge, then it can be augmented with two different points $a_{i_{1}}$ and $a_{i_{2}}$ such that $\mathrm{CH}\left(a_{\Delta^{1}}, \ldots, a_{\Delta^{i-1}}, a_{i_{1}}, a_{\Delta^{i+1}}, \ldots, a_{\Delta^{n}}\right)$ and $C H\left(a_{\Delta^{1}}, \ldots, a_{\Delta^{i-1}}, a_{i_{2}}, a_{\Delta^{i+1}}, \ldots, a_{\Delta^{n}}\right)$ are facets of $Y$. We call $a_{i_{1}}$ and $a_{i_{2}}$ augmenting points for the ridge.

(d) If boundary-simplex borders of two different facets are identical, e.g.,

$$
C H\left(a_{\Delta^{1}}, \ldots, a_{\Delta^{i-1}}, a_{\Delta^{i+1}}, \ldots, a_{\Delta^{n}}\right),
$$

then this is a ridge separating the two facets, and the corresponding augmenting points determine a unique facet $\mathrm{CH}\left(a_{\Delta^{\prime}}, \ldots, a_{\Delta^{\prime}}, \ldots, a_{\Delta^{n}}\right)$ each.

(e) Two adjacent ridges intersect in a so-called peak

$$
C H\left(a_{\Delta^{1}}, \ldots, a_{\Delta^{i-1}}, a_{\Delta^{i+1}}, \ldots, a_{\Delta^{j-1}}, a_{\Delta^{j+1}}, \ldots, a_{\Delta^{n}}\right)
$$

of dimension $n-3$ which is a border or side of each of the ridges.

\section{Some Famous Algorithmic Concepts}

Relying on the thesis of Dwyer [13, pp. 19-25], we briefly describe the most important algorithmical approaches. At a first glance it might be desirable to know the set of vertices in advance. This can be achieved by so-called preprocessing, and only afterward could the convex-hull construction on $\mathcal{V}$ instead of $\left\{a_{1}, \ldots, a_{m}\right\}$ be applied. Whether such a preprocessing is worthwhile, can be judged only for the single case and the special algorithm.

For each point $a_{i}$ we can decide whether it is a vertex of $Y$ by solving the following linear optimization problem:

minimize $\bar{a}^{T} x$

subject to $\quad\left(a_{j}-\bar{a}\right)^{T} x \leqq 1 \quad$ for $\quad j=1, \ldots, m, \quad j \neq i$, $\left(a_{i}-\bar{a}\right)^{T} x=1$,

where $\bar{a}$ stands for $(1 / m)\left(a_{1}+\cdots+a_{m}\right)$, i.e., $\bar{a}$ is the barycenter of $Y$.

If there are feasible points $x$, then $a_{i}$ must be a vertex ( $\bar{a}$ cannot be a vertex as long as the problem is nondegenerate).

So we have to solve an LP with $m$ restrictions and $n$ variables. From the work of Megiddo [23] and Clarkson [8] it is known that the worst-case complexity for such a 
problem is bounded by $O(m) \cdot C(n)$. Unfortunately, $C(n)$ will not be polynomial, on the other side the dependency on $m$ is linear. However, this should not induce the impression of fast solvability; notice the bad behavior of $C(n)$. On the other hand, the exclusion of the irrelevant $a_{i}$ 's may dramatically reduce the computation time for the pure convex-hull construction. Whether this is the case or not, is part of our study.

Now some examples for hull construction methods follow.

(a) Enumeration. Since there are $\left(\begin{array}{l}m \\ n\end{array}\right) n$-tuples of the type $\left(a_{\Delta^{\prime}}, \ldots, a_{\Delta^{n}}\right)$, all those candidates can be enumerated and checked whether they generate a facet or not. For this purpose the normal vector on the hyperplane induced by $a_{\Delta^{\prime}}, \ldots, a_{\Delta^{n}}$ is calculated. This hyperplane partitions $\mathbb{R}^{n}$ into two half-spaces. It is easy to check whether one of these two half-spaces contains all points $a_{1}, \ldots, a_{m}$. Exactly this would imply that $C H\left(a_{\Delta^{1}}, \ldots, a_{\Delta^{n}}\right)$ is a facet. This algorithm is extremely lengthy and time-wasting. Its worst-case complexity is $O\left(m^{n}\right)$.

(b) The Beneath-Beyond Algorithm of Kallay [21] and Seidel [24]. This is an incremental algorithm, which successively calculates the auxiliary polytopes $C H\left(a_{1}, \ldots, a_{l}\right)$ for $l \in\{n, \ldots, m\}$. Each iteration step uses information on $C H\left(a_{1}, \ldots, a_{l}\right)$, accepts $a_{l+1}$ as a new generator, and calculates $C H\left(a_{1}, \ldots, a_{l+1}\right)$. Here it is necessary to update files of facets and boundary-simplex borders ("ridges") for the actual polytope.

During one iteration step (from $\mathrm{CH}\left(a_{1}, \ldots, a_{l}\right)$ to $\mathrm{CH}\left(a_{1}, \ldots, a_{l+1}\right)$ ) we must decide which of the old facets remain, which disappear, and which enter now. Therefore we need information on the relative position of the new point $a_{l+1}$ with respect to the affine hull of each old facet. Since we look at a facet of $C H\left(a_{1}, \ldots, a_{l}\right)$, all points had been located in one of the two half-spaces. If $a_{l+1}$ belongs to the same half-space, then the old facet remains a facet after acceptance of $a_{l+1}$. Otherwise the old facet loses its property and has to be discarded. This decision can be interpreted as a visibility check. Facets are dropped if they are visible from $a_{l+1}$, else they are retained. In addition to the retained old facets, new facets will be introduced. For this purpose, determine all ridges which simultaneously belong to a visible and an invisible facet. These ridges form the "visibility horizon." Now augment each of these ridges (generated by $n-1$ points) with $a_{l+1}$. This augmentation delivers the new facets. The changes in the files of facets and ridges must be recorded.

This algorithm does not admit a simple analysis, because the facet sets in intermediate steps may vary dramatically. This depends strongly on the chosen order in which the points are accepted. In addition, the auxiliary polytopes $C H\left(a_{1}, \ldots, a_{l}\right)$ hardly resemble the final product $C H\left(a_{1}, \ldots, a_{m}\right)$.

(c) Random Sampling, by Clarkson and Shor [9], respectively Seidel [26]. A concept based on the Beneath-Beyond method, but accelerated by updating additional information, leads to an expected calculation time of $O\left(m^{\lfloor n / 2\rfloor}\right)$, when the order of accepting the points is randomly chosen.

Suppose, that $a_{1}, \ldots, a_{l}$ have been accepted and that the facial structure of 
$C H\left(a_{1}, \ldots, a_{l}\right)$ is completely available. In addition, the following information should be updated:

1. For each $a_{j}(j>l)$, a list asking: Which ridges of $C H\left(a_{1}, \ldots, a_{l}\right)$ are visible from $a_{j}$ ?

2. For each current ridge of the form $C H\left(a_{\Delta^{1}}, \ldots, a_{\Delta^{n-1}}\right)$, a list asking: For which $a_{j}$ 's $(j>l)$ is the ridge visible?

Having these lists (and handling them as a "conflict graph") makes the acceptance of $a_{l+1}$ rather easy. The completely visible facets and ridges bounding two visible facets are discarded. The invisible facets are retained. Horizon ridges induce new facets by augmentation with $a_{l+1}$. Horizon peaks are combined with $a_{l+1}$ and induce the new ridges. So far, we need only list 1 for $a_{l+1}$. To update both lists we have to erase the discarded ridges. For the old horizon ridges the visibility may have been lost, because they have a new incident facet. However, the elements of list 2 suffice for updating. For new ridges (resp. old horizon peaks) it is useful to know that the peak belongs to two horizon ridges of $a_{l+1}$. Checking list 2 for both gives a superset for the corresponding $a_{j}$ 's, from which the new ridge may be visible.

So the update effort depends on the number of elements in all lists of type 2 belonging to ridges which are in list 1 of $a_{l+1}$. However, here random sampling demonstrates its power. Elementary probability calculations show that for growing $l$ the single lists of type 1 or 2 tend to become empty (or to contain few elements with high probability). This fact is used to calculate a bound for the expected effort.

(d) The Shelling Algorithm of Seidel [25]. Suppose that an observer moves on the axis $\left\{x \mid x=\bar{a}+\lambda e_{n}, \lambda \in \mathbb{R}^{+}\right\}$with increasing $\lambda$ ( $\bar{a}$ is the barycenter of $Y, e_{n}=(0, \ldots, 0,1)$ is the "northern direction") and looks at $Y$. Then the facets on the "northern" hemisphere will become visible successively. If we-afterward-move in the opposite direction, i.e., with $\lambda \in \mathbb{R}^{-}$decreasing, we get an analogous sequence of facets in the southern hemisphere. Reversing the order of the second move and joining the first sequence with the reversed second sequence simulates a north-south movement of a "horizon" over $Y$ until all facets are discovered.

To realize this sequence mathematically, in a first stage the values of $\lambda$ (the times) where the single points $a_{i}$ will become visible have to be determined. These values $\bar{\lambda}_{i}$ are the solutions of the LPs:

maximize $\lambda$

$$
\text { subject to } \sum_{\substack{j=1 \\ j \neq i}}^{m} \rho_{j}\left(a_{j}-a_{i}\right)=\vec{a}+\lambda e_{n}-a_{i}, \quad \rho_{1}, \ldots, \rho_{m} \geqq 0, \quad \lambda \geqq 0 .
$$

The solution simultaneously tells us a facet becoming visible exactly with $a_{j}$. So we have a time-ordered sequence of some of the facets. However, we want to have the complete visibility sequence. Suppose that at a time $\bar{\lambda}$ all visible facets have been listed and that the ridges and the peaks of the actual horizon have been stored. Then we should know the next facet. There are two cases:

(1) either it is generated by $n$ points which are already visible;

(2) or the next facet is one of the special set which comes up with a new visible point. 
Every candidate for (1) results from joining two incident horizon ridges and their common peak to a "potential facet." For such a potential facet the visibility time is calculated by intersecting its affine hull with the axis $\left\{x \mid x=\bar{a}+\lambda e_{n}\right\}$. For (2) the visibility times are already known. Now it is essential for the shelling concept that among all the present candidates of (1) and (2) that one with the earliest visibility is chosen and put into our final sequence.

Then the three files of facets, ridges, and peaks have to be updated. This is easily done. \#F main iterations are necessary for discovering new facets. The update effort is less than \#F. $C_{1}(n) \cdot \ln m$, while the solution of the $m$ necessary LPs takes $m \cdot m \cdot C_{2}(n)$ steps.

One chance for saving would come from the knowledge and use of $\mathcal{V}$ in advance. This would replace $m^{2}$ by $(\# \mathcal{V})^{2}$ in the above formula. The key to such an improvement lies in preprocessing with a Divide-and-Conquer method due to Bentley and Shamos [3] for vertex enumeration. For that purpose the point-set has to be divided in two, the vertices of the convex hull for each half have to be determined, and which of those are even vertices of the convex hull of the whole set has to be decided via linear optimization. Applying this concept recursively becomes extremely profitable, if a considerable share of points becomes redundant.

We can observe the saving effect by looking at the order of the expected effort under certain stochastic models. Here shelling without preprocessing requires (as a function of $m$ ) an effort of

$$
O\left(m^{2}+E(\# \mathfrak{F}) \cdot \log m\right)
$$

and, with the help of preprocessing,

$$
O\left(m+E(\# \mathcal{V})^{2}+E(\# \mathfrak{F}) \log m\right) .
$$

For the distributions discussed later in this paper, we obtain the case of parameter $k$ for the Shelling Algorithm an expected $m$-dependency of

$$
\begin{array}{ll}
O(m) & \text { for } k>\frac{n-1}{2} \\
O(m \log m) & \text { for } k=\frac{n-1}{2}, \\
O\left(m^{2(n-1) /(2 k+n-1)}\right) & \text { for } k<\frac{n-1}{2},
\end{array}
$$

when we use preprocessing. Without preprocessing even the expected value stays at $O\left(m^{2}\right)$.

(e) The Gift-Wrapping Algorithm of Chand and Kapur [7]. Analogous statements apply for the Gift-Wrapping Algorithm, which is the subject of our study. This algorithm realizes a walk over the surface of $Y$ from facet to (adjacent) facet. Each facet change can be interpreted as crossing a ridge. Our goal is to discover all facets on this walk. However, this will not always work straightforwardly, because we may get into a dead end. That means that we arrive at a facet whose neighbors are all already known. Then 
we have to walk back the same way until a facet with at least one unknown neighbor is reached.

Again, the number of main iterations is $O(\# \mathfrak{F})$. Since a facet change crosses a ridge which belongs to both facets, we essentially replace one generator point by another generator. However, this substitute is unique. So for each of the $m-n$ remaining points it has to be checked whether it is the searched substitute. The updating effort for the necessary data-files amounts to $O(\# \mathfrak{F} \cdot \log (\# \mathfrak{F}))$ as a function of $m$ only. For the expected effort Dwyer [13] obtains statements like

$$
O(m \cdot E(\# \mathfrak{F})) \quad \text { for Gift-Wrapping without preprocessing }
$$

and

$$
O\left(m+E(\# \mathcal{V})^{2}+\mathrm{E}(\# \mathcal{V} \cdot \# \mathfrak{F})\right) \quad \text { with preprocessing. }
$$

Our aim is to calculate the expected complexity

- for a whole family of certain distributions over the unit ball, which is parametrized, and the result should show the dependency on that parameter,

- exactly also in its dependency upon $n$, the dimension of the space,

- for an algorithm avoiding preprocessing in order to proceed without the very bad influence of solving many LPs and without wasting time for a possibly useless job,

- showing that the success of preprocessing with respect to the $m$-dependency can also be achieved by our algorithm, which saves time implicitly.

Therefore we develop a rule of how to exclude many points from the substitute check for each individual facet. This is a kind of a Throw-Away Principle as introduced by Akl and Toussaint [1] and later by Devroye [10].

Finally we achieve the following main result.

Theorem 1. For rotation-symmetric, independent, and identical distribution of $m$ random points on an $n$-dimensional unit ball with arbitrary parameter $k \in(-1, \infty)$ and radial density function

$$
f_{k}(r)=\frac{\left(1-r^{2}\right)^{k} r^{n-1}}{\int_{0}^{1}\left(1-\tau^{2}\right)^{k} \tau^{n-1} d \tau}
$$

our combination of the Gift-Wrapping Algorithm with the Throw-Away Principle requires on the average not more than

$$
\text { Const } \begin{aligned}
\cdot & \left\{D_{0}(n, k)+m D_{1}(n, k)+m^{(n-1) /(n+1+2 k)}(\ln m) D_{2}(n, k)\right. \\
& +m^{1+(n-3-2 k) /(n+1+2 k)} D_{3}(n, k) \\
& \left.+m^{1-(2 k+2) /(n+1+2 k)}(\ln m)^{(2 k+2) /(n+1+2 k)+1} D_{4}(n, k)\right\}
\end{aligned}
$$

arithmetic operations for calculating all facets of $\mathrm{CH}\left(a_{1}, \ldots, a_{m}\right)$. The functions $D_{0}(n, k), \ldots, D_{4}(n, k)$ can be derived explicitly. 
(f) Gift-Wrapping Without Storage, by Avis and Fukuda [2]. Avis and Fukuda [2] developed a version of the Gift-Wrapping Algorithm which avoids most of the storage requirements. This can be achieved by switching to the equivalent vertex-enumeration problem for a polyhedron given by $m$ inequalities.

This polyhedron can be regarded as the feasible region of a linear programming problem. Suppose that for a chosen objective a unique optimal vertex exists (which can be achieved easily). Then we can start at every vertex and apply a fixed variant of the Simplex Algorithm (Bland's pivot rule) improving the objective, until the optimal vertex is reached.

The direction of walking on such a Simplex path depends on the current vertex, not on the start vertex. So, the set of all Simplex paths generated by that variant creates a tree with its root at the optimal vertex. This tree contains all vertices. However, this tree helps "Gift-Wrapping" to find its way. We simply run down that tree (starting from the root) with depth-first search. At each vertex we have to figure out which of the possible pivot steps are "reverse optimization steps." That means which pivot steps are done in reverse direction on an optimization path mentioned before. We decide which of the possible reverse Bland steps is to be realized by use of a lexicographic criterion. This information enables us to run down to the leaves of the tree. For going upward again, we follow the optimization path (leading the same way up) until we meet a parent vertex admitting other unused reverse optimization steps. The next unused step can easily be found as a consequence of Bland's pivot rule and of our lexicographic decision rule.

The advantage comes from the fact that we need not search for the possible unused steps in a file of vertices or edges. Hence we save the storage effort. The worst-case effort for that procedure turns out to be

$$
O(m \cdot n \cdot \# \mathfrak{F})
$$

\section{The Role of the Simplex Algorithm in a Walk over the Surface of $Y$}

Similarly to the procedure for solving linear optimization problems, the Simplex Algorithm can be used to organize a walk over the surface of $Y$, which eventually visits all facets of $Y$.

According to Lemma 1.1 every facet has exactly $n$ exits, each ridge stands for such an exit (resp. for the border between two facets). On pp. 69-95 of [4] we have described how to walk over successively adjacent boundary simplices, where each simplex change corresponds to a pivot step. If we intend to leave the facet $C H\left(a_{\Delta^{1}}, \ldots, a_{\Delta^{n}}\right)$ via the exit ridge $C H\left(a_{\Delta^{1}}, \ldots, a_{\Delta^{n-1}}\right)$, then there are $m-n$ candidates available for replacing $a_{\Delta^{n}}=: a_{i_{1}}$. Exactly one of them (without loss of generality $a_{i_{2}}$ ) is actually able to augment $\left(a_{\Delta^{\prime}}, \ldots, a_{\Delta^{n-1}}\right)$ in such a way that $C H\left(a_{\Delta^{1}}, \ldots, a_{\Delta^{n-1}}, a_{i_{2}}\right)$ becomes a facet or boundary simplex. This means that $a_{\Delta^{1}}, \ldots, a_{\Delta^{n-1}}, a_{i_{2}}$ span a hyperplane $H\left(a_{\Delta^{1}}, \ldots, a_{\Delta^{n-1}}, a_{i_{2}}\right)$, which divides $\mathbb{R}^{n}$ in such a way that all points $a_{1}, \ldots, a_{m}$ lie in one of the two closed half-spaces induced by $H$. If we interpret the linear independent vectors $a_{\Delta^{\prime}}, \ldots, a_{\Delta^{n}}$ as a basis of $\mathbb{R}^{n}$, then $a_{1}, \ldots, a_{m}$ as well as $e_{1}, \ldots, e_{n}$ are generated as linear combinations of the basis vectors.

We denote by $A_{\Delta}$ the submatrix of $A=\left(a_{1}, \ldots, a_{m}\right)$ consisting of the columns 
$a_{\Delta^{1}}, \ldots, a_{\Delta^{n}}$. Then the formulas

$$
\begin{aligned}
& \alpha_{1}=\left(A_{\Delta}\right)^{-1} a_{1}, \ldots, \alpha_{m}=\left(A_{\Delta}\right)^{-1} a_{m}, \\
& \gamma_{1}=\left(A_{\Delta}\right)^{-1} e_{1}, \ldots, \gamma_{n}=\left(A_{\Delta}\right)^{-1} e_{n}
\end{aligned}
$$

deliver the representation vectors of $a_{1}, \ldots, a_{m}$ (resp. $e_{1}, \ldots, e_{n}$ ) in the new coordinate system with respect to the basis $a_{\Delta^{\prime}}, \ldots, a_{\Delta^{n}}$. In addition, we get useful information by having the so-called slacks

$$
\begin{aligned}
\beta^{j} & =1-\alpha_{j}^{T}\left(A_{\Delta}^{T}\right)^{-1} \mathbb{1}=1-\alpha_{j}^{T} \mathbb{1} \\
-x^{i} & =0-e_{i}^{T}\left(A_{\Delta}^{T}\right)^{-1} 1=0-\gamma_{i}^{T} \mathbb{1} .
\end{aligned}
$$

Here $x=\left(x^{1}, \ldots, x^{n}\right)^{T}$ is the normal vector to $H\left(a_{\Delta^{1}}, \ldots, a_{\Delta^{n}}\right)$, which satisfies

$$
a_{\Delta^{\prime}}^{T} x=1, \ldots, a_{\Delta^{n}}^{T} x=1 .
$$

\begin{tabular}{|c|c|c|c|c|c|c|}
\hline & $a_{1}$ & $a_{j}$ & $a_{m}$ & $e_{1}$ & & $e_{n}$ \\
\hline$a_{\Delta^{\prime}}$ & $\vdots$ & $\vdots$ & $\vdots$ & $\vdots$ & & $\vdots$ \\
\hline : & : & : & : & : & & : \\
\hline$a_{\Delta^{\prime}}$ & $\alpha_{1}$ & $\alpha_{j}$ & $\alpha_{m}$ & $\gamma_{1}$ & $\left(A_{\Delta}\right)^{-1}$ & $\gamma_{n}$ \\
\hline$\vdots$ & $\vdots$ & $\vdots$ & $\vdots$ & $\vdots$ & & $\vdots$ \\
\hline$a_{\Delta^{\prime \prime}}$ & $\vdots$ & $\vdots$ & $\vdots$ & $\vdots$ & & $\vdots$ \\
\hline & $\beta^{1}$ & $\beta^{j}$ & $\beta^{m}$ & $-x^{1}$ & $\cdots$ & $-x^{n}$ \\
\hline
\end{tabular}

These data can be stored in the Simplex tableau belonging to the boundary simplex $C H\left(a_{\Delta^{1}}, \ldots, a_{\Delta^{\prime \prime}}\right)$, which looks as follows:

If $a_{\Delta^{i}}=a_{i_{1}}$ is to be replaced in the basis, then we have to find a substitute $a_{i_{2}}$ with $i_{2} \notin \Delta$. Using $a_{i_{2}}$, we obtain a hyperplane $H\left(a_{\Delta^{1}}, \ldots, a_{\Delta^{i-1}}, a_{i_{2}}, a_{\Delta^{i+1}}, \ldots, a_{\Delta^{n}}\right)$, whose normal vector $x$ satisfies

$$
a_{i}^{T} x \leqq 1, \quad \forall i=1, \ldots, m, \quad \text { or } \quad a_{i}^{T} x \geqq 1, \quad \forall i=1, \ldots, m .
$$

That means that in the new tableau it is required that

$$
\beta^{j} \geqq 0, \quad \forall j=1, \ldots, m, \quad \text { or } \quad \beta^{j} \leqq 0, \quad \forall j=1, \ldots, m .
$$

The Simplex method recognizes the vector $a_{i_{2}}$ as the extremal argument among the $m-n$ candidates for the so-called quotient criterion. The geometrical task in determining $a_{i_{2}}$ is simply: Find that point $a_{j}(j \notin \Delta)$ that realizes (by augmentation to $\left.a_{\Delta^{1}}, \ldots, a_{\Delta^{i-1}}, a_{\Delta^{i+1}}, \ldots, a_{\Delta^{\prime \prime}}\right)$ the smallest rotation angle with respect to

$$
C H\left(a_{\Delta^{\prime}}, \ldots, a_{\Delta^{i-1}}, a_{i_{1}}, a_{\Delta^{i+1}}, \ldots, a_{\Delta^{n}}\right) .
$$

Depending on the configuration before the pivot step, we have to decide according to the following rules. We use $\hat{\beta}^{j}$ for denoting the slack after the pivot operation (basis exchange). 
In general the formula for updating the slacks is $\hat{\beta}^{j}=\beta^{j}-\left(\beta^{t} \cdot \alpha_{j}^{i}\right) / \alpha_{t}^{i}$ when $a_{\Delta^{i}}=a_{i_{1}}$ is substituted by $a_{t}$.

(i) If $\beta \geqq 0$ completely and if there are entries $\alpha_{j}^{i}<0$, then determine a $t$ with $\alpha_{t}^{i}<0$, such that $\beta^{t} / \alpha_{t}^{i} \geqq \beta^{j} / \alpha_{j}^{i}$ for all $j$ with $\alpha_{j}^{i}<0$. Then we have $\hat{\beta}^{j}=$ $\beta^{j}-\beta^{t} \alpha_{j}^{i} / \alpha_{t}^{i} \geqq 0$ for those $j$ 's, and for the $j$ 's with $\alpha_{j}^{i} \geqq 0$ anyway.

(ii) If $\beta \geqq 0$ and if there are no entries $\alpha_{j}^{i}<0$, then determine a $t$ with $\alpha_{t}^{i}>0$, such that $\beta^{t} / \alpha_{t}^{i} \geqq \beta^{j} / \alpha_{j}^{i}$ for all $j$ with $\alpha_{j}^{i}>0$. Then we have $\hat{\beta}^{j}=\beta^{j}-\beta^{t} \alpha_{j}^{i} / \alpha_{t}^{i} \leqq 0$ for all those $j$ 's, and for the $j$ 's with $\alpha_{j}^{i} \leqq 0$ anyway.

(iii) If $\beta \leqq 0$ and if there are entries $\alpha_{j}^{i}<0$, then determine a $t$ with $\alpha_{1}^{i}<0$, such that $\beta^{t} / \alpha_{t}^{i} \leqq \beta^{j} / \alpha^{j}$ for all $j$ with $\alpha_{j}^{i}<0$. Then we have $\hat{\beta}^{j}=\beta^{j}-\beta^{t} \alpha_{j}^{i} / \alpha_{t}^{i} \leqq 0$ for all those $j$ 's, and for the $j$ 's with $\alpha_{j}^{i} \geqq 0$ anyway.

(iv) If $\beta \leqq 0$ and if there are no entries $\alpha_{j}^{i}<0$, then determine a $t$ with $\alpha_{t}^{i}>0$, such that $\beta^{t} / \alpha_{t}^{i}<\beta^{j} / \alpha_{j}^{i}$ for all $j$ with $\alpha_{j}^{i}>0$. Then we have $\hat{\beta}^{j}=\beta^{j}-\beta^{t} \alpha_{j}^{i} / \alpha_{t}^{i} \geqq 0$ for all those $j$ 's, and for the $j$ 's with $\alpha_{j}^{i} \leqq 0$ anyway.

Definition 2.1. In the case of $\beta^{1}, \ldots, \beta^{m} \geqq 0$, i.e., $a_{1}^{T} x \leqq 1, \ldots, a_{m}^{T} x \leqq 1$, we call $C H\left(a_{\Delta^{1}}, \ldots, a_{\Delta^{n}}\right)$ a facet or boundary simplex of the first kind. In the case of $\beta^{1}, \ldots, \beta^{m} \leqq 0$, i.e., $a_{1}^{T} x \geqq 1, \ldots, a_{m}^{T} x \geqq 1$, we call $C H\left(a_{\Delta^{\prime}}, \ldots, a_{\Delta^{\prime \prime}}\right)$ a facet or boundary simplex of the second kind. $\mathfrak{F}$ can be partitioned into $\mathfrak{F}_{1}$ and $\mathfrak{F}_{2}$, which are the sets of facets of the first and second kind.

Remark. A boundary simplex of the first kind has the property that its affine hull (the hyperplane through the generating points) puts all remaining points and the origin in the same half-space. A boundary simplex of the second kind has the property that its affine hull separates the origin from all other remaining points (Fig. 1).

Remark. Application of the four-case quotient criterion mentioned above corresponds to minimizing the rotation angle around the axis $A H\left(a_{\Delta^{1}}, \ldots, a_{\Delta^{i-1}}, a_{\Delta^{i+1}}, \ldots, a_{\Delta^{\prime \prime}}\right)$

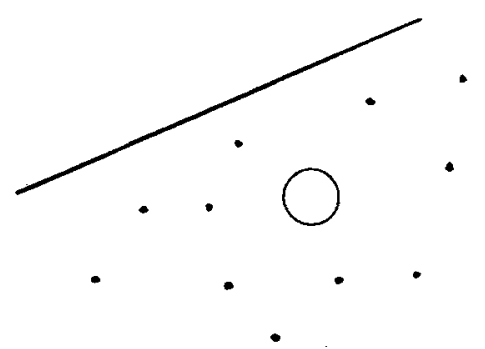

$1^{\text {st }}$ kind

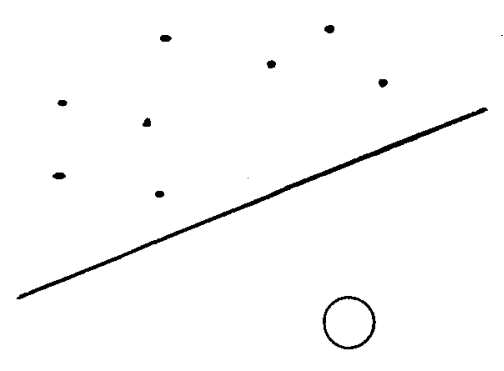

$2^{\text {nd }}$ kind

Fig. 1 


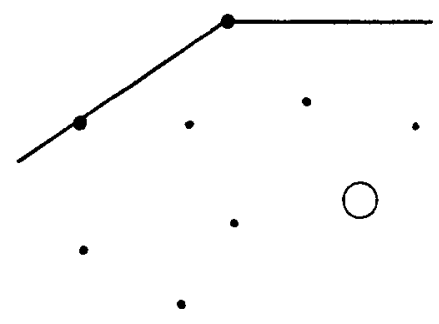

1 st kind

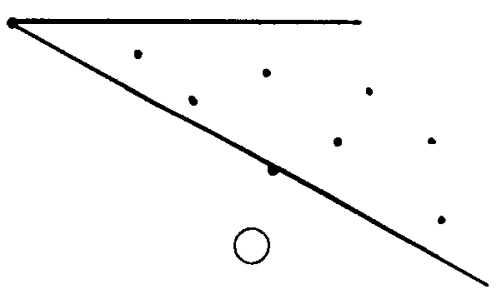

2nd kind

Fig. 2

( $A H=$ affine hull). Starting from the hyperplane $H\left(a_{\Delta^{1}}, \ldots, a_{\Delta^{i-1}}, a_{\Delta^{i}}, a_{\Delta^{i+1}}, \ldots, a_{\Delta^{n}}\right)$ we rotate until we meet the first point replacing $a_{\Delta^{i}}$ (called $a_{i_{2}}$ ). This can also be interpreted as cracking the surface at the rotation axis until we get another supporting point. The quotient criterion delivers that first supporting point (Fig. 2).

Remark. For an efficient implementation it is only necessary to update $A_{\Delta}^{-1}$ and $x$ permanently and to generate the tableau row for $a_{\Delta^{i}}$ and the $\beta$ row. This can be done with one scalar product per entry. The total effort for the quotient criterion of one pivot step is therefore $O(m n)$.

\section{A Simple Gift-Wrapping Algorithm}

We present an algorithm, which discovers all facets.

Definition 3.1. A ridge or border of a facet is called saturated if both augmentation points $a_{i_{1}}$ and $a_{i_{2}}$ are known and hence the two incident facets are discovered. It is called unsaturated, if only one augmentation point is known, the other unknown. That means that we do not know and did not visit the other facet so far. It is called undiscovered, if no incident facet is known.

Definition 3.2 (Simple Gift Wrapping).

Initialization

(a) Determine a start facet as follows:

(i) Augment the set $\left\{a_{1}, \ldots, a_{m}\right\}$ by $\rho e_{1}, \ldots, \rho e_{n}(\rho>0)$ with $\rho>\left\|a_{i}\right\|_{1}$ for all $i=1, \ldots, m$.

(ii) Eliminate successively the vectors $\rho e_{i}$ from the basis by pivot steps and by entering respective facets of the rest system.

(iii) After $n$ such exchange steps (eliminated $\rho e_{i}$ 's are not available for entering), we have a start-boundary simplex of the original system in hand.

(b) Store the obtained facet in a tree-storing file. 
(c) Note all borders of the given facet in a lexicographically organized tree file together with the corresponding augmentation point in a file for unsaturated facet borders.

Typical Step

(1) Search for an unsaturated ridge to the current facet. If there is one, go directly to (4).

(2) Carry out reverse pivot steps according to the stored facets in the tree file until you have a facet with at least one unsaturated ridge. If there is one, go to (4).

(3) Stop, since the unsaturated facet-borders file is empty.

(4) Leave the current facet across the unsaturated border after determination of the second augmentation point $a_{i_{2}}$, i.e., carry out a forward pivot step to discover a new facet. Note the new facet in the facet file.

(5) Determine all ridges to the current facet. For each of them do the following:

(i) Check whether this ridge is already in the unsaturated facet-borders file.

(ii) If yes, then erase the ridge, because it is now saturated, since we have already seen it from the second incident facet.

(iii) If no, then store the ridge in the unsaturated facet-borders file, since we have seen it the first time.

(6) Go to (1).

Theorem 2. The Gift-Wrapping Algorithm determines and discovers all facets.

Proof. Each forward pivot step enters a new facet, since we are traversing an unsaturated boundary-simplex border (ridge). The number of facets is bounded by $\left(\begin{array}{c}m \\ n\end{array}\right)$, hence the number of forward pivot steps is finite. The algorithm stops only if no unsaturated ridge is known. Then there cannot be any unvisited facet. This is because the facet set seen as a graph is connected. If there was an unvisited facet, then we would have a path for a pivot-step walk from the current facet to that unknown facet. On this path at least one unsaturated facet border must be traversed. This contradicts the potential existence of an unknown facet when the algorithm stops.

Theorem 3. The simple Gift-Wrapping Algorithm requires an arithmetic effort of not more than

$$
C\left\{(\# \mathfrak{F}+n)\left[n m+n^{2} \ln m\right]\right\},
$$

where $C$ is a constant.

Proof. In the initialization there are $n$, and in the main process there are \#F, forward pivot steps without any variation. The only interesting question is the work which has to be done in the single pivot step.

Each updating of $A_{\Delta}^{-1}$ requires $n^{2}$ arithmetic operations. At most $m-n$ quotients have to be calculated, for each one, two entries have to be reproduced, which costs $2 \cdot 2 n$ arithmetic operations. 
Searching for an observed ridge in the tree of unsaturated ridges can be done in $O(n \ln m)$ as well as inserting and/or erasing these ridges. This relies on the fact that the whole tree cannot have more than $\left(\begin{array}{c}m \\ n\end{array}\right)$ leaves.

The number of backtracking pivot steps is less than the number of forward pivot steps, because they lead backward from a dead end to a facet visited before with an unsaturated border. This happens exactly on a subpath which had been used before in the forward direction. In addition, a backward step will never be repeated, since leaving a facet without unsaturated borders backward means that we will never visit this facet again.

The consequence of these arguments is an upper bound of

$$
O\left((\# \mathfrak{F}+n)\left[n m+n^{2} \ln m\right]\right\} .
$$

Some Remarks on Data Storing. We store all the unsaturated ridges (each one with its additional augmentation point) in a search tree in the following way.

$$
\left(a_{\Delta^{1}}, \ldots, a_{\Delta^{i-1}}, a_{\Delta^{i+1}}, \ldots, a_{\Delta^{n}} \mid a_{i_{1}}\right)
$$

may be the information which is to be saved in pointer or path form. In our tree we find the following nodes for our purpose:

$\begin{array}{ll}(0) \text { in the highest hierarchical rank } & 0 \\ \left(a_{\Delta^{\prime}}\right) \text { in rank } & 1 \\ \left(a_{\Delta^{\prime}}, a_{\Delta^{2}}\right) \text { in rank } & 2 \\ \vdots & \vdots \\ \left(a_{\Delta^{\prime}}, \ldots, a_{\Delta^{\prime}}\right) \text { in rank } & l(l<i) \\ \vdots & \vdots \\ \left(a_{\Delta^{\prime}}, \ldots, a_{\Delta^{i-1}}\right) \text { in rank } & i-1 \\ \left(a_{\Delta^{\prime}}, \ldots, a_{\Delta^{i-1}}, a_{\Delta^{i+1}}\right) \text { in rank } & i \\ \vdots & \vdots \\ \left(a_{\Delta^{\prime}}, \ldots, a_{\Delta^{i-1}}, a_{\Delta^{i+1}}, \ldots, a_{\Delta^{l}}\right) \text { in rank } & l-1(l>i) \\ \vdots & \vdots \\ \left(a_{\Delta^{\prime}}, \ldots, a_{\Delta^{i-1}}, a_{\Delta^{i+1}}, \ldots, a_{\Delta^{n}}\right) \text { in rank } & n-1 \\ \left(a_{\Delta^{\prime}}, \ldots, a_{\Delta^{i-1}}, a_{\Delta^{i+1}}, \ldots, a_{\Delta^{n}} \mid a_{i_{1}}\right) \text { in rank } & n\end{array}$

To find a leave of that tree, one has to decide between (up to) $m$ candidates in each stage (step from one rank to another). Note that $\Delta^{1}<\Delta^{2}<\cdots<\Delta^{n}$ always. In an efficient implementation this single decision can also be organized in a binary tree structure. So one of the above decisions can be replaced by $\ln m$ binary decisions. Therefore the whole search requires $O(n \ln m)$ decisions. The same holds for the insertion and erasure of paths. 


\section{Accelerating the Gift-Wrapping Algorithm}

In the general case not all $m$ points may be vertices of $Y$. In this case they "disappear" in the interior of $Y$. We know that

$$
\begin{gathered}
\mathcal{V}=\left\{a_{i} \mid a_{i} \text { vertex of } C H\left(a_{1}, \ldots, a_{m}\right)\right\} \subset\left\{a_{1}, \ldots, a_{m}\right\} \\
\text { but } C H(\mathcal{V})=C H\left(a_{1}, \ldots, a_{m}\right) .
\end{gathered}
$$

If a subset of the points is already capable of generating the complete convex hull, then saving effects seem possible. It would be fine if we could replace the factor $m$ by a lower number, hopefully by the number of vertices \#V . However, how should we realize the appropriate algorithm and how should we distinguish vertices from other points in advance?

We briefly recall the distinction between facets of the first and second kind. A facet is of the first kind if its affine hull bounds one half-space containing the origin and all remaining points. It is of the second kind if the corresponding half-space again contains all points, but not the origin.

Now we present a concept for savings while determining the more frequently occurring facets of the first kind. First, we introduce some necessary notation.

Definition 4.1. Corresponding to a simplex $C H\left(a_{\Delta^{\prime}}, \ldots, a_{\Delta^{n}}\right)$, define $H\left(a_{\Delta^{\prime}}, \ldots, a_{\Delta^{n}}\right)$ as the hyperplane containing the complete simplex and define $h\left(a_{\Delta^{\prime}}, \ldots, a_{\Delta^{n}}\right)$ as its height, i.e., the distance between the origin and $H\left(a_{\Delta^{1}}, \ldots, a_{\Delta^{n}}\right)$.

The following auxiliary result helps to exclude certain points from participation in the process of determining a certain facet.

\section{Lemma 4.1.}

(a) If $\left\|a_{\Delta^{\prime}}\right\|<\bar{h}$ for a simplex $C H\left(a_{\Delta^{\prime}}, \ldots, a_{\Delta^{\prime}}, \ldots, a_{\Delta^{n}}\right)$, then $h\left(a_{\Delta^{\prime}}, \ldots, a_{\Delta^{n}}\right)<$ $\bar{h}$.

(b) All simplices with $h\left(a_{\Delta^{\prime}}, \ldots, a_{\Delta^{n}}\right) \geqq \bar{h}$ are generated only by points $a_{\Delta^{i}}$ such that $\left\|a_{\Delta^{i}}\right\| \geqq \bar{h}$.

(c) Let $C H\left(a_{\Delta^{\prime}}, \ldots, a_{\Delta^{\prime \prime}}\right)$ be a boundary simplex of the first kind for $C H\left(a_{1}, \ldots, a_{l}\right)$ $(l<m)$ and let $\left\|a_{l+1}\right\|, \ldots,\left\|a_{m}\right\|<h\left(a_{\Delta^{\prime}}, \ldots, a_{\Delta^{n}}\right)$. Then $C H\left(a_{\Delta^{\prime}}, \ldots, a_{\Delta^{n}}\right)$ is a boundary simplex of $\mathrm{CH}\left(a_{1}, \ldots, a_{m}\right)$ as well.

(d) If the quotient criterion predicts a new boundary simplex of the first kind for $C H\left(a_{\Delta^{\prime}}, \ldots, a_{\Delta^{n}}\right)$ after evaluation of the points $a_{1}, \ldots, a_{l}$, and if simultaneously $\left\|a_{i+1}\right\|, \ldots,\left\|a_{m}\right\| \leqq h\left(a_{\Delta^{1}}, \ldots, a_{\Delta^{n}}\right)$, the prediction is correct, and $a_{l+1}, \ldots, a_{m}$ need not be checked further.

Proof. (a) and (b) are opposite formulations of the same insight, which is based on the fact that $\left\|a_{\Delta^{i}}\right\| \geqq h\left(a_{\Delta^{1}}, \ldots, a_{\Delta^{n}}\right)$ for all $i=1, \ldots, n$. (c) holds, because the points $a_{l+1}, \ldots, a_{m}$ cannot question the property of being a boundary simplex. (d) is simply the algorithmic consequence from (c). 
Motivated by that lemma we prefer to sort the points according to their Euclidean norm (in decreasing order). After that we apply the quotient criterion to the points in distance-decreasing order. As soon as the distance of the point under consideration becomes smaller than the height of the existing boundary simplex, we can stop, because the boundary simplex is now fixed. The remaining quotient calculations become superfluous and useless. Since it may not be necessary to sort all points, we can (for further acceleration) implement a heap for dynamical sorting according to the norm. We sort only as far as necessary.

Definition 4.2. We denote the set of already sorted points by $\mathcal{S}$. These are the \#S vectors of greatest Euclidean length. For a certain height value $h$, denote by $s(h)$ the number of points satisfying $\left\|a_{i}\right\| \geqq h$.

Now we are able to formulate an accelerated version of our algorithm.

Definition 4.3 (Accelerated Gift-Wrapping Algorithm).

Initialization

(a) Implement a heap for sorting points dynamically according to their length in decreasing order.

(b) Determine a start facet as follows (as before in Definition 3.2):

(i) Augment the set $\left\{a_{1}, \ldots, a_{m}\right\}$ by $\rho e_{1}, \ldots, \rho e_{n}(\rho>0)$ with $\rho>\left\|a_{i}\right\|_{1}$ for all $i=1, \ldots, m$. Now $C H\left(\rho e_{1}, \ldots, \rho e_{n}\right)$ is a facet of

$$
C H\left(a_{1}, \ldots, a_{m}, \rho e_{1}, \ldots, \rho e_{n}\right) .
$$

(ii) Eliminate successively the vectors $\rho e_{i}$ from the basis by pivot steps and by entering respective facets of the rest system.

(iii) After $n$ such exchange steps (eliminated $\rho e_{i}$ 's are not available for entering), we have a start-boundary simplex of the original system at hand.

(c) Store the obtained facet in a tree-storing file.

(d) Note all borders of the given facet in a lexicographically organized tree file together with the corresponding augmentation point in a file for unsaturated facet borders.

Typical Step

(1) Search for an unsaturated ridge to the current facet. If there is one, go directly to (4).

(2) Carry out reverse pivot steps according to the stored facets in the tree file until you have a facet with at least one unsaturated ridge. If there is one, go to (4).

(3) Stop, since the unsaturated facet-borders file is empty.

(4) Leave the current facet across the unsaturated border after determination of the second augmentation point $a_{i_{2}}$. For that purpose do the following for $j=1, \ldots, m$ starting from $j=1$ :

(i) Determine the $j$-longest vector (new $a_{j}$ ) either from the list of sorted points or by using the heap; repeat with $j=j+1$ if $a_{j} \in \Delta$.

(ii) Apply the quotient criterion and determine the current optimum and the preliminary substitute $\tilde{a}_{i_{2}}$. 
(iii) If $C H\left(a_{\Delta^{1}}, \ldots, a_{\Delta^{i-1}}, \tilde{a}_{i_{2}}, a_{\Delta^{i+1}}, \ldots, a_{\Delta^{n}}\right)$ is of the second kind, then set $j=j+1$ and go to (i).

(iv) If $C H\left(a_{\Delta^{\prime}}, \ldots, a_{\Delta^{i-1}}, \tilde{a}_{i_{2}}, a_{\Delta^{i+1}}, \ldots, a_{\Delta^{\prime \prime}}\right)$ is of the first kind, then check whether $h\left(a_{\Delta^{\prime}}, \ldots, a_{\Delta^{i-1}}, \tilde{a}_{i_{2}}, a_{\Delta^{i+1}}, \ldots, a_{\Delta^{n}}\right) \leqq\left\|a_{j}\right\|$. If yes and $j<m$, set $j=j+1$ and go to (i).

Note the new facet in the facet file.

(5) Determine all ridges to the current facet. For each of them do the following:

(i) Check whether this ridge is already in the unsaturated borders file.

(ii) If yes, then erase the ridge, because it is saturated now, since we have seen it from the second facet.

(iii) If no, then store the ridge in the unsaturated facet-borders file, since we have seen it for the first time.

(6) Go to (1).

Theorem 4. The accelerated algorithm discovers all facets.

Proof. Essentially, we have changed only (4). Here we refer to Lemma 4.1. This allows us to dispense with calculating quotients, as soon as we have a preliminary facet of certain height $h$ and as all following points satisfy $\left\|a_{j}\right\| \leqq h$.

The sorting process is continued if necessary in (4(i)).

Theorem 5. The total effort for the accelerated algorithm is

$$
\begin{gathered}
O\left\{n\left[n^{2}+m n+n^{2} \ln m\right]+\sum_{B S \in \mathfrak{F}_{1}}\left[n^{2}+s\left(h_{B S}\right) n+n^{2} \ln m\right]\right. \\
\left.+\sum_{B S \in \mathfrak{F}_{2}}\left[m n+n^{2} \ln m\right]+m+(\ln m) s\left(\operatorname{Min} \geqq 0 h_{\mathfrak{F}}\right)\right\} .
\end{gathered}
$$

Here $h_{B S}$ is the actual height of the required facet $(B S)$ and $s\left(h_{B S}\right)$ is the number of points with greater Euclidean length. In addition we use

$$
\operatorname{Min}{ }^{\geq 0} h_{\mathfrak{F}}:=\left\{\begin{array}{lll}
\operatorname{Min}_{B S \in \mathfrak{F}_{1}} h_{B S} & \text { if } & \mathfrak{F}_{2}=\emptyset \\
0 & \text { if } & \mathfrak{F}_{2} \neq \emptyset
\end{array}\right.
$$

Proof. The proof is as before, but in the situation of a boundary simplex of the first kind we need only $s\left(h_{B S}\right)$ instead of $m-n$ quotients. Also new is the requirement to calculate the height of the preliminary boundary simplex. However, this can also be done in $O(n)$ time per point such that the order mentioned above is not affected. $O(m)$ time is now required for implementation of the heap and the last term (for sorting as many points as required) is also new. 


\section{A Probabilistic Model}

Now we present a family of distributions which serve as a basis for the probabilistic considerations.

Definition 5.1. Let $a_{1}, \ldots, a_{m}$ be distributed symmetrically under rotations, identically and independently over the $n$-dimensional unit ball (without origin).

Remark. Such a distribution model for our points satisfies our condition of nondegeneracy (1.1), with probability 1 .

Definition 5.2. A rotation-symmetric distribution can be characterized uniquely by its "radial distribution function":

$$
F(r):=P(\|x\| \leqq r) \quad \text { for } \quad r \in[0, \infty),
$$

where $P(\|x\| \leqq r)$ denotes the probability that a random point $x$ is of Euclidean length not greater than $r$.

In our family of distributions over the unit ball it is clear that $F(r)=1$ always for $r \geqq 1$. If the radial distribution function has a density, then we denote it by $f(r)$, i.e.,

$$
F(r)=\int_{0}^{r} f(\rho) d \rho \quad \text { for all } \quad r \in[0, \infty) .
$$

If the distribution over $\mathbb{R}^{n}$ with distribution function $\hat{F}$ has a density $\hat{f}$, then

$$
F(r):=\lambda_{n-1}\left(\omega_{n}\right) \int_{0}^{r} t^{n-1} \tilde{f}(t) d t \quad \text { where } \quad \tilde{f}(t)=f(t) \cdot \frac{1}{t^{n-1} \cdot \lambda_{n-1}\left(\omega_{n}\right)} .
$$

Here $\tilde{f}:[0, \infty) \rightarrow \mathbb{R}$ is a function such that $\hat{f}(x)=\tilde{f}(\|x\|)$ for all $x \in \mathbb{R}^{n}$, and $\hat{f}$ is a density over $\mathbb{R}^{n}$, i.e., $\hat{f}: \mathbb{R}^{n} \rightarrow \mathbb{R}^{+}$with $\hat{f}\left(x_{1}\right)=\hat{f}\left(x_{2}\right)$ for all $x_{1}, x_{2}$ with $\left\|x_{1}\right\|=\left\|x_{2}\right\|$.

Definition 5.3 (Notation for Balls). $\omega_{n}$ denotes the $n$-dimensional unit sphere $\left\{x \in \mathbb{R}^{n} \mid\|x\|=1\right\}$ and $\Omega_{n}$ denotes the $n$-dimensional unit ball $\left\{x \in \mathbb{R}^{n} \mid\|x\| \leqq 1\right\}$. $\lambda_{k}(\cdot)$ stands for the $k$-dimensional Lebesgue measure of the specified set, hence

$$
\lambda_{n-1}\left(\omega_{n}\right)=\frac{2 \pi^{n / 2}}{\Gamma(n / 2)} \quad \text { and } \quad \lambda_{n}\left(\Omega_{n}\right)=\frac{\pi^{n / 2}}{\Gamma((n+2) / 2)} .
$$

In this paper we restrict discussion to a special class of distributions over $\Omega_{n}$, which is particularly suitable for the necessary integration operations and - on the other handdemonstrates all typical features of changing the weight or mass of the distribution from the boundary (sphere) of the ball to the center (origin) of the ball.

Definition 5.4. Under variation of a parameter $k \in(-1, \infty)$ we define

$$
F_{k}(r):= \begin{cases}\frac{\int_{0}^{r}\left(1-\tau^{2}\right)^{k} \tau^{n-1} d \tau}{\int_{0}^{1}\left(1-\tau^{2}\right)^{k} \tau^{n-1} d \tau} & \text { for } 0 \leqq r \leqq 1, \\ 1 & \text { for } r>1 .\end{cases}
$$


These radial distributions have corresponding radial densities, namely,

$$
f_{k}(r):=\frac{\left(1-r^{2}\right)^{k} r^{n-1}}{\int_{0}^{1}\left(1-\tau^{2}\right)^{k} \tau^{n-1} d \tau} \quad \text { for } \quad 0 \leqq r \leqq 1 \quad \text { and } 0 \text { elsewhere. }
$$

Remark. The parameter $k$ gives the weighting on the radii between 0 and 1 . $k \rightarrow-1$ means extremal weight at the boundary of the ball, and increasing $k$ describes an increment of weight in the interior and a decrease at the sphere.

Exploiting the relation (5.2) we obtain

$$
f(r)=\lambda_{n-1}\left(\omega_{n}\right) r^{n-1} \hat{f}(x) \quad \text { for all } x \text { with } \quad\|x\|=r
$$

and, for our special case with $k$,

$$
\hat{f}(x)=\frac{f(\|x\|)}{\lambda_{n-1}\left(\omega_{n}\right)\|x\|^{n-1}}=\frac{\left(1-\|x\|^{2}\right)^{k}}{\lambda_{n-1}\left(\omega_{n}\right) \int_{0}^{1}\left(1-\tau^{2}\right)^{k} \tau^{n-1} d \tau} .
$$

Remark. Interesting special cases are:

$$
\begin{aligned}
& k=0 \Rightarrow \hat{f} \text { constant on } \Omega_{n} \hat{=} \text { uniform distribution on } \Omega_{n}, \\
& k \rightarrow-1 \Rightarrow \text { extremal dominance at } r=1 \hat{=} \text { uniform distribution on } \omega_{n}, \\
& k \rightarrow \infty \Rightarrow \text { extremal dominance at } r=0 \hat{=} \text { totally centralized, } \\
& k=\frac{n-1}{2} \Rightarrow \text { radial density symmetric around } r=\frac{1}{2} .
\end{aligned}
$$

Equipped with these tools we calculate the necessary expected values corresponding to Theorem 5. However, first we specialize the formulas mentioned above to our special cases.

We use the following substitution rule.

\section{Lemma 5.1.}

(a)

$$
\begin{aligned}
\int_{0}^{1}\left(1-\tau^{2}\right)^{k} \tau^{n-1} d \tau & \left.=\int_{0}^{1}\left(1-\tau^{2}\right)^{k} \tau \cdot \tau^{n-2} d \tau \quad \text { (by substitution } u=\tau^{2}\right) \\
& =\frac{1}{2} \int_{0}^{1}(1-u)^{k} u^{(n-2) / 2} d u=\frac{\Gamma(k+1) \Gamma(n / 2)}{2 \Gamma(k+1+n / 2)}
\end{aligned}
$$

where $\Gamma(\cdot)$ denotes the well-known Gamma function.

(b)

$$
\begin{gathered}
\int_{h}^{1}\left(r^{2}-h^{2}\right)^{l}\left(1-r^{2}\right)^{k} r d r \quad\left(\text { by substitution } u=\frac{r^{2}-h^{2}}{1-h^{2}}\right) \\
=\int_{0}^{1} u^{l}\left(1-h^{2}\right)^{l}(1-u)^{k}\left(1-h^{2}\right)^{k}\left(1-h^{2}\right) \cdot \frac{1}{2} d u
\end{gathered}
$$




$$
\begin{aligned}
& =\frac{1}{2}\left(1-h^{2}\right)^{l+k+1} \int_{0}^{1} u^{l}(1-u)^{k} d u \\
& =\frac{1}{2}\left(1-h^{2}\right)^{l+k+1} \frac{\Gamma(k+1) \Gamma(l+1)}{\Gamma(k+l+2)} .
\end{aligned}
$$

More detailed explanations concerning technical details can be obtained from Appendices 6.1 and 6.2 (pp. 245-255) of [4].

Now we formulate our special versions of functions with parameter $k$, which appears as the subscript (resp. second subscript) index.

Definition 5.5. For these distributions we can also study the marginal distribution and the marginal density. The marginal distribution function is defined by

$$
G:[-1,1] \rightarrow[0,1] \text { and } G(h):=P\left(x^{n} \leqq h\right) .
$$

The marginal density function is defined by

$$
g:[-1,1] \rightarrow(0, \infty) \text { and } \int_{-1}^{h} g(\mathfrak{h}) d \mathfrak{h}=G(h)
$$

For general rotation-symmetric distributions the following marginal distribution and related functions are useful for our purposes:

$$
\begin{gathered}
G(h)=1-\frac{\lambda_{n-2}\left(\omega_{n-1}\right)}{\lambda_{n-1}\left(\omega_{n}\right)} \int_{h}^{1} \int_{h / r}^{1}\left(1-\sigma^{2}\right)^{(n-3) / 2} d \sigma d F(r) \\
g_{0}(h):=g(h)=\frac{\lambda_{n-2}\left(\omega_{n-1}\right)}{\lambda_{n-1}\left(\omega_{n}\right)} \int_{h}^{1} \frac{\left(r^{2}-h^{2}\right)^{(n-3) / 2}}{r^{n-2}} d F(r), \\
g_{1}(h):=g_{0}(h) \cdot E\left(\left|x^{1}\right| / x^{n}=h\right)=\frac{2 \lambda_{n-3}\left(\omega_{n-2}\right)}{(n-2) \lambda_{n-1}\left(\omega_{n}\right)} \int_{h}^{1} \frac{\left(r^{2}-h^{2}\right)^{(n-2) / 2}}{r^{n-2}} d F(r) \\
=\frac{1}{\pi} \int_{h}^{1} \frac{\left(r^{2}-h^{2}\right)^{(n-2) / 2}}{r^{n-2}} d F(r), \\
g_{2}(h):=g_{0}(h) \cdot E\left(\left(x^{1}\right)^{2} / x^{n}=h\right)=\frac{\lambda_{n-2}\left(\omega_{n-1}\right)}{(n-1) \lambda_{n-1}\left(\omega_{n}\right)} \int_{h}^{1} \frac{\left(r^{2}-h^{2}\right)^{(n-1) / 2}}{r^{n-2}} d F(r) .
\end{gathered}
$$

Then (5.11)-(5.14) specialize to

$$
\begin{aligned}
& G_{k}(h)=1-\frac{\lambda_{n-2}\left(\omega_{n-1}\right)}{\lambda_{n-1}\left(\omega_{n}\right)} \int_{h}^{1} \int_{h / r}^{1}\left(1-\sigma^{2}\right)^{(n-3) / 2} d \sigma\left(1-r^{2}\right)^{k} r^{n-1} d r \cdot \frac{2 \Gamma(k+1+n / 2)}{\Gamma(k+1) \Gamma(n / 2)} \\
& g_{0, k}(h)=\frac{\lambda_{n-2}\left(\omega_{n-1}\right)}{\lambda_{n-1}\left(\omega_{n}\right)} \cdot \frac{2 \Gamma(k+1+n / 2)}{\Gamma(k+1) \Gamma(n / 2)} \int_{h}^{1} \frac{\left(r^{2}-h^{2}\right)^{(n-3) / 2}}{r^{n-2}}\left(1-r^{2}\right)^{k} r^{n-1} d r \\
& =\frac{\Gamma(k+1+n / 2)}{\sqrt{\pi} \Gamma(k+1+(n-1) / 2)} \cdot\left(1-h^{2}\right)^{(n-1) / 2+k},
\end{aligned}
$$




$$
\begin{gathered}
g_{1, k}(h)=\frac{1}{\pi} \int_{h}^{1} \frac{\left(r^{2}-h^{2}\right)^{(n-2) / 2}}{r^{n-2}}\left(1-r^{2}\right)^{k} r^{n-1} d r \cdot \frac{2 \Gamma(k+1+n / 2)}{\Gamma(k+1) \Gamma(n / 2)} \\
=\frac{1}{\pi}\left(1-h^{2}\right)^{n / 2+k}, \\
g_{2, k}(h)=\frac{\lambda_{n-2}\left(\omega_{n-1}\right)}{(n-1) \lambda_{n-1}\left(\omega_{n}\right)} \int_{h}^{1} \frac{\left(r^{2}-h^{2}\right)^{(n-1) / 2}}{r^{n-2}}\left(1-r^{2}\right)^{k} r^{n-1} d r \cdot \frac{2 \Gamma(k+1+n / 2)}{\Gamma(k+1) \Gamma(n / 2)} \\
=\frac{\Gamma(k+1+n / 2)}{2 \sqrt{\pi} \Gamma(k+1+(n+1) / 2)}\left(1-h^{2}\right)^{(n+1) / 2+k}
\end{gathered}
$$

\section{The Expected Complexity for Our Algorithm}

We recall our upper bound for the total effort (Theorem 5):

$$
\begin{aligned}
& O\left\{n\left[n^{2}+m n+n^{2} \ln m\right]+\sum_{B S \in \mathfrak{F}_{1}}\left[n^{2}+s\left(h_{B S}\right) n+n^{2} \ln m\right]\right. \\
& \left.+\sum_{B S \in \mathfrak{F}_{2}}\left[m n+n^{2} \ln m\right]+m+(\ln m) s\left(\operatorname{Min}^{\geqq 0} h_{\mathfrak{F}}\right)\right\} .
\end{aligned}
$$

For achieving an upper bound for the expected total effort, we need the expected value of that bound. Since constant dimension factors can be treated trivially, only the following expectation values are interesting:

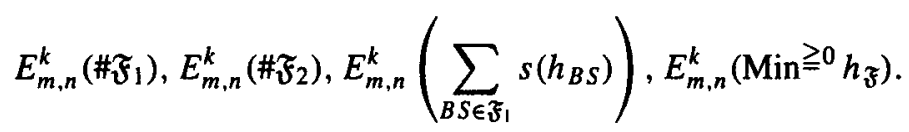

Here $E_{m, n}^{k}$ stands for the expected value for $m$ points, dimension $n$, and parameter $k$. These expectation values will now be calculated. We rely on the methods and techniques developed on pp. 245-255 of [4] for dealing with these types of integrals.

In general we exploit the linearity of expectation values. That means we count the potential candidates for having a certain property and multiply this number with the probability that a typical candidate actually satisfies the conditions. Here we can make use of the assumptions on identical and independent distributions. This general approach delivers the expected total numbers.

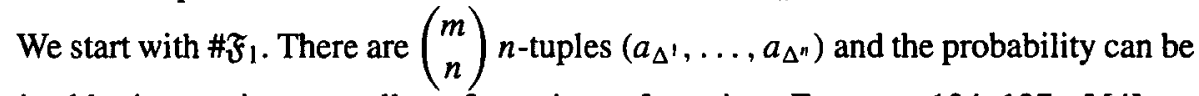
derived by integrating over all configurations of $n$ points. From pp. 134-137 of [4] we know that

$$
\begin{aligned}
E_{m, n}^{k}\left(\# \mathfrak{F}_{1}\right)= & \left(\begin{array}{c}
m \\
n
\end{array}\right) \int_{\mathbb{R}^{n}} \cdots \int_{\mathbb{R}^{n}} G\left(h\left(a_{1}, \ldots, a_{n}\right)\right)^{m-n} d \hat{F}\left(a_{1}\right) \cdots d \hat{F}\left(a_{n}\right) \\
= & \left(\begin{array}{c}
m \\
n
\end{array}\right) \lambda_{n-1}\left(\omega_{n}\right) \int_{0}^{1} G(h)^{m-n} \\
& \cdot \int_{\mathbb{R}^{n-1}} \cdots \int_{\mathbb{R}^{n-1}}|\operatorname{Det} B| \hat{f}\left(b_{1}\right) \cdots \hat{f}\left(b_{n}\right) d \bar{b}_{1} \cdots d \bar{b}_{n} d h,
\end{aligned}
$$


where

$$
\begin{aligned}
B & =\left[\begin{array}{ccc}
b_{1}^{1} & \cdots & b_{n}^{1} \\
\vdots & & \vdots \\
b_{1}^{n-1} & b_{n}^{n-1} \\
1 & 1
\end{array}\right] \\
& =\left(\begin{array}{l}
m \\
n
\end{array}\right) \lambda_{n-1}\left(\omega_{n}\right) \int_{0}^{1} G(h)^{m-n} \Lambda_{1}(h) d h,
\end{aligned}
$$

with

$$
\Lambda_{1}(h):=\int_{\mathbb{R}^{n-1}} \cdots \int_{\mathbb{R}^{n-1}}|\operatorname{Det} B| \hat{f}\left(b_{1}\right) \cdots \hat{f}\left(b_{n}\right) d \bar{b}_{1} \cdots d \bar{b}_{n} .
$$

The Cauchy-Schwartz inequality enables us to get a simpler upper bound for $\Lambda_{1}(h)$ by use of the functions $\Lambda_{0}(h)$ and $\Lambda_{2}(h)$ (see pp. 188-195 of [4]):

$$
\begin{gathered}
\Lambda_{0}(h):=\int_{\mathbb{R}^{n-1}} \cdots \int_{\mathbb{R}^{n-1}} \hat{f}\left(b_{1}\right) \cdots \hat{f}\left(b_{n}\right) d \bar{b}_{1} \cdots d \bar{b}_{n}, \\
\Lambda_{2}(h):=\int_{\mathbb{R}^{n-1}} \cdots \int_{\mathbb{R}^{n-1}}|\operatorname{Det} B|^{2} \hat{f}\left(b_{1}\right) \cdots \hat{f}\left(b_{n}\right) d \bar{b}_{1} \cdots d \bar{b}_{n} .
\end{gathered}
$$

Then it is clear that

$$
\Lambda_{\mathrm{I}}(h) \leq\left[\Lambda_{0}(h) \Lambda_{2}(h)\right]^{1 / 2} .
$$

$\Lambda_{1}(h)$ exists, since our distribution has bounded support. From pp. 188-194 of [4] we know that

$$
\begin{aligned}
& \Lambda_{0}(h)=g_{0}(h)^{n}, \\
& \Lambda_{2}(h)=n ! g_{2}(h)^{n-1} g_{0}(h) .
\end{aligned}
$$

Consequently,

$$
\Lambda_{\mathrm{l}}(h) \leqq\left[\Lambda_{0}(h) \Lambda_{2}(h)\right]^{1 / 2}=[n !]^{1 / 2} g_{0}(h)^{n} \cdot\left[\frac{g_{2}(h)}{g_{0}(h)}\right]^{(n-1) / 2} .
$$

Specialization delivers, according to (5.16) and (5.18),

$$
\begin{aligned}
\Lambda_{1, k}(h) \leqq & {[n !]^{1 / 2} g_{0, k}(h) \cdot g_{0, k}(h)^{(n-1) / 2} \cdot\left[\frac{1}{2(k+1+(n-1) / 2)}\left(1-h^{2}\right)\right]^{(n-1) / 2} } \\
= & {[n !]^{1 / 2} g_{0, k}(h) } \\
& \cdot\left(\frac{1}{2 \pi}\right)^{(n-1) / 2} \frac{\Gamma(k+1+n / 2)^{n-1}}{\Gamma(k+1+(n-1) / 2)^{n-1}(k+1+(n-1) / 2)^{(n-1) / 2}} \\
& \cdot\left(1-h^{2}\right)^{[n / 2+k](n-1)} .
\end{aligned}
$$


So we get

$$
\begin{aligned}
E_{m, n}^{k}\left(\# \mathfrak{F}_{1}\right)= & \left(\begin{array}{c}
m \\
n
\end{array}\right) \lambda_{n-1}\left(\omega_{n}\right) \int_{0}^{1} G_{k}(h)^{m-n} g_{0, k}(h)\left(1-h^{2}\right)^{[n / 2+k](n-1)} d h \\
& \cdot[n !]^{1 / 2}\left(\frac{1}{2 \pi}\right)^{(n-1) / 2} \\
& \cdot \frac{\Gamma(k+1+n / 2)^{n-1}}{\Gamma(k+1+(n-1) / 2)^{n-1}(k+1+(n-1) / 2)^{(n-1) / 2}} .
\end{aligned}
$$

Now there is a relation between $G_{k}(h)$ and $\left(1-h^{2}\right)$, namely,

$$
\begin{aligned}
1-G_{k}(h) & =\int_{h}^{1} g_{0, k}(\mathfrak{h}) d \mathfrak{h}=\frac{\Gamma(k+1+n / 2)}{\sqrt{\pi} \Gamma(k+1+(n-1) / 2)} \cdot \int_{h}^{1}\left(1-\mathfrak{h}^{2}\right)^{(n-1) / 2+k} d \mathfrak{h} \\
& \geqq \frac{\Gamma(k+1+n / 2)}{\sqrt{\pi} \Gamma(k+1+(n-1) / 2)} \cdot \int_{h}^{1}\left(1-\mathfrak{h}^{2}\right)^{(n-1) / 2+k} \mathfrak{h} d \mathfrak{h} \\
& =\frac{\Gamma(k+1+n / 2)}{\sqrt{\pi} \Gamma(k+1+(n+1) / 2)} \frac{1}{2}\left(1-h^{2}\right)^{(n+1) / 2+k}
\end{aligned}
$$

Since the upper bound for $\Lambda_{1, k}(h)$ from (6.8) appearing in (6.9) is essentially a power of $\left(1-h^{2}\right)$, we can substitute it by something like a power of $1-G_{k}(h)$.

$$
\begin{aligned}
E_{m, n}^{k}\left(\#\left(\mathfrak{F}_{1}\right)\right) \leqq & \left(\begin{array}{c}
m \\
n
\end{array}\right) \lambda_{n-1}\left(\omega_{n}\right) \int_{0}^{1} G_{k}(h)^{m-n} g_{0, k}(h) \\
& \cdot\left(1-G_{k}(h)\right)^{([n / 2+k](n-1)) /((n+1) / 2+k)} d h \\
& \cdot[n !]^{1 / 2}\left(\frac{1}{2 \pi}\right)^{(n-1) / 2} \\
& \cdot \frac{\Gamma(k+1+n / 2)^{n-1}}{\Gamma(k+1+(n-1) / 2)^{n-1}(k+1+(n-1) / 2)^{(n-1) / 2}} \\
& \cdot\left[\frac{\Gamma(k+1+n / 2)}{\sqrt{\pi} \Gamma(k+1+(n+1) / 2)} \frac{1}{2}\right]^{-([n / 2+k](n-1)) /((n+1) / 2+k)} \\
= & \left(\begin{array}{l}
m \\
n
\end{array}\right) \lambda_{n-1}\left(\omega_{n}\right)[n !]^{1 / 2}\left(\frac{1}{2 \pi}\right)^{(n-1) / 2} \\
& \cdot\left[\frac{n+1+2 k}{k+1+(n-1) / 2}\right]^{(n-1) / 2} \pi^{(n-1) / 2} \\
& \cdot\left[\frac{2 \sqrt{\pi} \Gamma(k+1+(n+1) / 2)}{\Gamma(k+1+n / 2)}\right]^{-(n-1) /(n+1+2 k)} \cdot(n+1+2 k)^{(n-1) / 2} \\
& \cdot \int_{1 / 2}^{1} G^{m-n}(1-G)^{n-1-((n-1) /(n+1+2 k))} d G \quad(\text { see pp. 247-248 of [4]) } \\
\leqq & \frac{1}{n} \cdot m^{(n-1) /(n+1+2 k)} 2 \pi^{n / 2} \frac{[n !]^{1 / 2}}{\Gamma(n / 2)}(n+1+2 k)^{(n-1) / 2} \\
& \cdot\left[\frac{\Gamma(k+1+n / 2)}{2 \sqrt{\pi} \Gamma(k+1+(n+1) / 2)}\right]^{(n-1) /(n+1+2 k)}
\end{aligned}
$$


Remark. Using the Stirling formula, $E_{m, n}^{k}\left(\# \mathfrak{F}_{1}\right)$ can be approximated as follows:

$$
\begin{aligned}
E_{m, n}^{k}\left(\# \mathfrak{F}_{1}\right) \approx & \frac{1}{n} m^{(n-1) /(n+1+2 k)} 2^{n / 2} \pi^{n / 2} \cdot n^{1 / 4}(n+1+2 k)^{(n-1) / 2} \\
& \cdot\left(k+1+\frac{n}{2}\right)^{-(1 / 2) \cdot((n-1) /(n+1+2 k))} \cdot \text { Const . }
\end{aligned}
$$

Analogously we can estimate $E_{m, n}^{k}\left(\# \mathfrak{F}_{2}\right)$. Here we have to study the situation where all points lie beyond $H$ :

$$
\begin{aligned}
E_{m, n}^{k}\left(\# \mathfrak{F}_{2}\right)= & \left(\begin{array}{l}
m \\
n
\end{array}\right) \lambda_{n-1}\left(\omega_{n}\right) \int_{\mathbb{R}^{n}} \cdots \int_{\mathbb{R}^{n}}\left[1-G\left(h\left(a_{1}, \ldots, a_{n}\right)\right)\right]^{m-n} d \hat{F}\left(a_{1}\right) \cdots d \hat{F}\left(a_{n}\right) \\
\leq & \left(\begin{array}{l}
m \\
n
\end{array}\right) \lambda_{n-1}\left(\omega_{n}\right) \int_{0}^{1}[1-G(h)]^{m-n} \Lambda_{1}(h) d h \\
& (\text { in our special case }) \\
= & \left(\begin{array}{l}
m \\
n
\end{array}\right) \lambda_{n-1}\left(\omega_{n}\right)[n !]^{1 / 2}[n+1+2 k]^{(n-1) / 2} \\
& \cdot\left[\frac{\Gamma(k+1+n / 2)}{2 \sqrt{\pi} \Gamma(k+1+(n+1) / 2)}\right]^{(n-1) /(n+1+2 k)} \\
& \cdot \int_{1 / 2}^{1}(1-G)^{m-n+(n-1)-((n-1) /(n+1+2 k))} d G \\
= & \left(\begin{array}{l}
m \\
n
\end{array}\right) \frac{2 \pi^{n / 2}}{\Gamma(n / 2)}[n !]^{1 / 2}[n+1+2 k]^{(n-1) / 2} \\
& \cdot\left[\frac{\Gamma(k+1+n / 2)}{2 \sqrt{\pi} \Gamma(k+1+(n+1) / 2)}\right]^{(n-1) /(n+1+2 k)} \\
& \cdot \frac{1}{m-(n-1) /(n+1+2 k)} \cdot \frac{1}{2}
\end{aligned}
$$

This expression tends quickly to 0 when $n$ is fixed and $m$ increases.

Remark. From [29] it is known that the probability of having any facets of the second kind is

$$
\frac{1}{2}^{m-1} \sum_{k=0}^{n-1}\left(\begin{array}{c}
m-1 \\
k
\end{array}\right) .
$$

If we multiply this probability with $\left(\begin{array}{l}m \\ n\end{array}\right)$, the worst possible number of candidates, then we obtain a worse bound on $E_{m, n}^{k}\left(\# \mathfrak{F}_{2}\right)$. However, even this bound proves the asymptotic unimportance of second-kind facets.

Now we proceed to the random variable $\sum_{B S \in \mathfrak{F}_{1}} s\left(h_{B S}\right)$. The philosophy here is as follows. We want to know how many points have to be checked in the total process. For each facet which is to be determined, the set of checked points may differ. So for each 
facet we count the number of checks and summarize. Formally this can be done in the following way. We enumerate all $\left(\begin{array}{l}m \\ n\end{array}\right)$ simplices, determine their heights, and count all points not belonging to $\Delta$, which have a Euclidean length greater than $h$. However, then we ignore this result if the simplex does not have the facet property. So our candidate enumeration works as follows: We have $\left(\begin{array}{c}m \\ n\end{array}\right)$ simplices of the type $C H\left(a_{\Delta^{\prime}}, \ldots, a_{\Delta^{n}}\right)$ and we have $m-n$ additional points $a_{j}(j \notin \Delta)$, which could be checked. So there are $\left(\begin{array}{l}m \\ n\end{array}\right)(m-n)$ combinations. The combination must be counted if the simplex is a boundary simplex and if $\left\|a_{j}\right\| \geqq h$. For formalizing that count decision, we rely on indicator functions:

$$
\begin{aligned}
& E_{m, n}^{k}\left(\sum_{B S \in \mathfrak{F}_{1}} s\left(h_{B S}\right)\right) \\
&=\left(\begin{array}{l}
m \\
n
\end{array}\right)(m-n) \\
& \cdot P\left(C H\left(a_{\Delta^{\prime}}, \ldots, a_{\Delta^{n}}\right) \text { is facet of the first kind and }\left\|a_{j}\right\| \geqq h\left(a_{1}, \ldots, a_{n}\right)\right) \\
&=\left(\begin{array}{l}
m \\
n
\end{array}\right)(m-n) \int_{\mathbb{R}^{n}} \ldots \int_{\mathbb{R}^{n}} G\left(h\left(a_{1}, \ldots, a_{n}\right)\right)^{m-n-1} \\
& \cdot I_{a_{j} \text { below } H\left(a_{1}, \ldots, a_{n}\right) \text { and }\left\|a_{j}\right\| \geqq h\left(a_{1}, \ldots, a_{n}\right)}\left(a_{j}, h\left(a_{1}, \ldots, a_{n}\right), H\left(a_{1}, \ldots, a_{n}\right)\right) \\
& \cdot d \hat{F}\left(a_{j}\right) d \hat{F}\left(a_{1}\right) \cdots d \hat{F}\left(a_{n}\right) .
\end{aligned}
$$

The integral becomes much simpler if we increase the right-hand side (by a factor at most 2) through dropping the condition " $a_{j}$ below $H\left(a_{1}, \ldots, a_{n}\right)$ ":

$$
\begin{aligned}
E_{m, n}^{k}\left(\sum_{B S \in \mathfrak{F}_{1}} s\left(h_{B S}\right)\right) \leqq & \left(\begin{array}{c}
m \\
n
\end{array}\right)(m-n) \int_{\mathbb{R}^{n}} \cdots \int_{\mathbb{R}^{n}} G\left(h\left(a_{1}, \ldots, a_{n}\right)\right)^{m-n-1} \\
& \cdot I_{\left\|a_{j}\right\| \geqq h}\left(a_{j}, h\right) \cdot d \hat{F}\left(a_{j}\right) d \hat{F}\left(a_{1}\right) \cdots d \hat{F}\left(a_{n}\right) .
\end{aligned}
$$

With the methods of Borgwardt [4, pp. 134-137], we get

$$
=\left(\begin{array}{l}
m \\
n
\end{array}\right)(m-n) \lambda_{n-1}\left(\omega_{n}\right) \int_{0}^{1} G(h)^{m-n} \Lambda_{1}(h)(1-F(h)) d h .
$$

Only the factor $1-F(h)$ is new, which specializes to

$$
1-F_{k}(h)=\frac{\int_{h}^{1}\left(1-\tau^{2}\right)^{k} \tau^{n-1} d \tau}{\int_{0}^{1}\left(1-\tau^{2}\right)^{k} \tau^{n-1} d \tau} \leqq \frac{(1 / 2(k+1))\left(1-h^{2}\right)^{k+1}}{\frac{\Gamma(k+1) \Gamma(n / 2)}{2 \Gamma(k+1+n / 2)}}
$$

because

$$
\int_{h}^{1}\left(1-\tau^{2}\right)^{k} \tau^{n-1} d \tau \leqq \int_{h}^{1}\left(1-\tau^{2}\right)^{k} \tau d \tau=\frac{1}{2} \frac{1}{k+1}\left(1-h^{2}\right)^{k+1} .
$$


Insertion into (6.16) delivers

$$
\begin{aligned}
& E_{m, n}^{k}\left(\sum_{B S \in \mathfrak{F}_{\mathfrak{T}}} s\left(h_{B S}\right)\right) \leqq\left(\begin{array}{l}
m \\
n
\end{array}\right)(m-n) \lambda_{n-1}\left(\omega_{n}\right) \int_{0}^{1} G_{k}(h)^{m-n-1} g_{0, k}(h) \\
& \cdot\left(1-h^{2}\right)^{[n / 2+k](n-1)+k+1} d h \cdot[n !]^{1 / 2}\left(\frac{1}{2 \pi}\right)^{(n-1) / 2} \\
& \Gamma(k+1+n / 2)^{n-1} \\
& \cdot \overline{\Gamma(k+1+(n-1) / 2)^{n-1}(k+1+(n-1) / 2)^{(n-1) / 2}} \\
& \frac{\Gamma(k+1+n / 2)}{\Gamma(k+2) \Gamma(n / 2)} \\
& \leqq\left(\begin{array}{c}
m \\
n
\end{array}\right)(m-n) \lambda_{n-1}\left(\omega_{n}\right) \\
& \cdot \int_{0}^{1} G_{k}(h)^{m-n-1} g_{0, k}(h)\left[1-G_{k}(h)\right]^{n-1+(2 k+3-n) /(n+1+2 k)} d h \\
& \cdot[n !]^{1 / 2}\left(\frac{1}{2 \pi}\right)^{(n-1) / 2} \\
& \frac{\Gamma(k+1+n / 2)^{n-1}}{\Gamma(k+1+(n-1) / 2)^{n-1}(k+1+(n-1) / 2)^{(n-1) / 2}} \\
& \frac{\Gamma(k+1+n / 2)}{\Gamma(k+2) \Gamma(n / 2)} \\
& \cdot\left[\frac{2 \sqrt{\pi} \Gamma(k+1+(n+1) / 2)}{\Gamma(k+1+n / 2)}\right]^{n-1+(2 k+3-n) /(n+1+2 k)} \\
& =\left(\begin{array}{c}
m \\
n
\end{array}\right)(m-n) \lambda_{n-1}\left(\omega_{n}\right) \\
& \int_{1 / 2}^{1} G^{m-n-1}[1-G]^{n-1+(2 k+3-n) /(n+1+2 k)} d G \\
& \cdot[n !]^{1 / 2} 2^{(n-1) / 2}\left(\frac{n+1}{2}+k\right)^{(n-1) / 2} \frac{\Gamma(k+1+n / 2)}{\Gamma(k+2) \Gamma(n / 2)} \\
& \cdot\left[\frac{2 \sqrt{\pi} \Gamma(k+1+(n+1) / 2)}{\Gamma(k+1+n / 2)}\right]^{(2 k+3-n) /(n+1+2 k)}
\end{aligned}
$$

Again, we obtain an upper bound for the integral from pp. 247-248 of [4]. Here we distinguish the cases where $\delta=(n-3-2 k) /(n+1+2 k)$ is positive or negative:

(1)

$$
\begin{aligned}
\left(\begin{array}{l}
m \\
n
\end{array}\right)(m-n) \int_{0}^{1} G^{m-n-1}[1-G]^{n-1-\delta} d G & \leqq(m-n) \cdot \frac{1}{n}\left(\frac{m+1-\delta}{n-1}+k\right)^{\delta} \\
& \leqq\left(\frac{1}{n-1}\right)^{1+\delta}(m+1)^{1+\delta} \quad(\delta \text { positive })
\end{aligned}
$$


(2)

$$
\begin{aligned}
\left(\begin{array}{c}
m \\
n
\end{array}\right)(m & -n) \int_{0}^{1} G^{m-n-1}[1-G]^{n-1-\delta} d G \\
& =(n+1)\left(\begin{array}{c}
m \\
n+1
\end{array}\right) \int_{0}^{1} G^{m-(n+1)}[1-G]^{n-(1+\delta)} d G \\
& \leqq\left(\frac{m+1-(1+\delta)}{n}\right)^{(1+\delta)} \\
& \leqq\left(\frac{m+1}{n}\right)^{1-|\delta|} \quad(\delta \text { negative }) .
\end{aligned}
$$

Hence we have a common upper bound of $(1 /(n-1))^{1+\delta}(m+1)^{1+\delta}$. Insertion yields

$$
\begin{aligned}
E_{m, n}^{k}\left(\sum_{B S \in \mathfrak{F}_{1}} s\left(h_{B S}\right)\right) \leq & (m+1)^{1+(n-3-2 k) /(n+1+2 k)}\left(\frac{1}{n-1}\right)^{1+(n-3-2 k) /(n+1+2 k)} \\
& \cdot \frac{2 \pi^{n / 2}}{\Gamma(n / 2)}[n !]^{1 / 2} 2^{(n-1) / 2}\left(\frac{n+1}{2}+k\right)^{(n-1) / 2} \frac{\Gamma(k+1+n / 2)}{\Gamma(k+2) \Gamma(n / 2)} \\
& \cdot\left[\frac{2 \sqrt{\pi} \Gamma(k+1+(n+1) / 2)}{\Gamma(k+1+n / 2)}\right]^{(2 k+3-n) /(n+1+2 k)}
\end{aligned}
$$

Now we arrive at the last expectation value, namely, the number of points which have to be sorted. Remember that in the case of nonexistence of facets of the second kind a point needs to be in the subset of sorted points only, if there is at least one facet of the first kind, whose height is smaller than the Euclidean length of the point. Since it requires $\ln m$ time to determine the next greatest element of a set, when a heap is installed, the total effort for sorting the critical subset is $(\ln m) s\left(\operatorname{Min} \geqq 0 h_{\mathfrak{F}}\right.$ ), where Min ${ }^{\geq 0} h_{\mathfrak{F}}$ describes the minimal height of a facet of $Y$, and where facets of the second kind are given "height 0. ."

In order to obtain a practicable estimation, we must use a seemingly crude method. For an appropriate $\bar{h}(m, n, k)$ we suggest that all points with $\left\|a_{i}\right\|>\bar{h}$ are sorted anyway. $\mathrm{Min} \geqq 0 h_{\mathfrak{F}}$ is a very complicated random variable. Its distribution is hardly computable. Hence we estimate in the remaining area as follows:

$$
E\left(\#\left\{a_{i} \mid \operatorname{Min} \geqq 0 h_{\mathcal{F}}<\left\|a_{i}\right\|<\bar{h}\right\}\right) \leqq E\left(\#\left\{B S \mid h_{B S}<\bar{h}\right\}\right) \cdot(m-n) .
$$

Here we count a point as often as there is a facet of smaller height than $\bar{h}$. However, this delivers an upper bound:

$$
E_{m, n}^{k}\left(s\left(\operatorname{Min} \geqq 0 h_{\mathfrak{F}}\right)\right) \leqq m[1-F(\bar{h})]+\left(\begin{array}{l}
m \\
n
\end{array}\right)(m-n) \lambda_{n-1}\left(\omega_{n}\right) \int_{0}^{\bar{h}} G(h)^{m-n} \Lambda_{1}(h) d h .
$$

Since we know that

$$
\lambda_{n-1}\left(\omega_{n}\right) \int_{0}^{1} \Lambda_{1}(h) d h=1 \quad\left(\# \mathcal{F}_{1} \text { when } m=n\right)
$$


we can conclude that

$$
\begin{aligned}
\left(\begin{array}{c}
m \\
n
\end{array}\right)(m-n) \lambda_{n-1}\left(\omega_{n}\right) \int_{0}^{\bar{h}} G(h)^{m-n} \Lambda_{1}(h) d h \\
\leqq \\
\quad\left(\begin{array}{c}
m \\
n
\end{array}\right)(m-n) G(\bar{h})^{m-n} \lambda_{n-1}\left(\omega_{n}\right) \int_{0}^{1} \Lambda_{1}(h) d h \\
\leqq m^{n+1} G(\bar{h})^{m-n} .
\end{aligned}
$$

Now we try to find an $\bar{h}$, which simultaneously keeps both summands from (6.21) relatively small. We consider the case where $m \gg n$. Then we choose $\bar{h}$ such that

$$
\left(1-\bar{h}^{2}\right)=\left[\frac{\ln m}{m-n} \sqrt{\pi}(n+1)(n+1+2 k)\right]^{1 /((n+1) / 2+k)}
$$

(This becomes $<1$ for sufficiently great $m$.) Then (compare (6.17)) the first summand satisfies

$$
\begin{aligned}
m\left[1-F_{k}(\bar{h})\right] \leqq & \frac{\Gamma(k+1+n / 2)}{\Gamma(k+2) \Gamma(n / 2)} m \\
& \cdot\left[\frac{\ln m}{(m-n)} \sqrt{\pi}(n+1)(n+1+2 k)\right]^{(k+1) /((n+1) / 2+k)}
\end{aligned}
$$

For the second summand, bounded by (6.23), we use (6.10):

$$
G_{k}(\bar{h}) \leqq 1-\frac{\Gamma(k+1+n / 2)}{2 \sqrt{\pi} \Gamma(k+1+(n+1) / 2)}\left(1-\bar{h}^{2}\right)^{(n+1) / 2+k} .
$$

Then

$$
\begin{aligned}
m^{n+1} G(\bar{h})^{m-n} \leqq & e^{(\ln m)(n+1)} \\
& \cdot\left[1-\frac{\Gamma(k+1+n / 2)}{2 \sqrt{\pi} \Gamma(k+1+(n+1) / 2)}\right. \\
& \left.\cdot\left(\frac{\ln m}{(m-n)} \sqrt{\pi}(n+1)(n+1+2 k)\right)^{((n+1) / 2+k) /((n+1) / 2+k)}\right]^{m-n} \\
\leqq & e^{(\ln m)(n+1)} \exp \left\{-\ln m(n+1) \cdot \frac{\Gamma(k+1+(n+2) / 2)}{\Gamma(k+1+(n+1) / 2)}\right\} \\
= & \exp \left\{\ln m(n+1) \cdot\left[1-\frac{\Gamma(k+1+(n+2) / 2)}{\Gamma(k+1+(n+1) / 2)}\right]\right\} \leqq 1
\end{aligned}
$$

So (6.25) delivers an upper bound for the order of the effort for sorting

$$
\begin{aligned}
(\ln m) m & \cdot\left(\frac{1}{m-n}\right)^{(k+1) /((n+1) / 2+k)}[\ln m \sqrt{\pi}(n+1)(n+1+2 k)]^{(k+1) /((n+1) / 2+k)} \\
& \frac{\Gamma(k+1+n / 2)}{\Gamma(k+2) \Gamma(n / 2)} \quad \text { in the case } m \gg n .
\end{aligned}
$$

This shows that the effort for sorting remains linear in $m$ in the asymptotical case. 


\section{The Main Results}

We summarize all our expectation values, insert them in Theorem 5, and describe the total effort.

Theorem 6. Let $a_{1}, \ldots, a_{m}$ be distributed independently, identically, and symmetrically under rotations with the radial density specified by the parameter $k \in(-1, \infty)$ :

$$
f_{k}(r)=\frac{\left(1-r^{2}\right)^{k} r^{n-1}}{\int_{0}^{1}\left(1-\tau^{2}\right)^{k} \tau^{n-1} d \tau} \quad \text { for } 0 \leqq r \leqq 1 \text { and } 0 \text { for } r>1 \text {. }
$$

Then the average number of arithmetic operations for determining the convex hull can be estimated from above by

$$
\begin{aligned}
C\{ & n^{3}+n^{2} m+n^{3} \ln m+n^{2} C_{1}(n, k) m^{(n-1) /(n+1+2 k)} \cdot(1+\ln m) \\
& +n C_{2}(n, k)(m+1)^{1+(n-3-2 k) /(n+1+2 k)} \\
& +n C_{3}(n, k)\left(\begin{array}{c}
m \\
n
\end{array}\right) \frac{m+n \ln m}{m-(n-1) /(n+1+2 k)} \frac{1}{2}^{m-(n-1) /(n+1+2 k)} \\
& \left.+m+(\ln m) m^{*}\right\} .
\end{aligned}
$$

All these figures $C_{i}(n, k)$ can be given explicitly:

$$
\begin{aligned}
C_{1}(n, k)= & \frac{1}{n} 2 \pi^{n / 2} \frac{[n !]^{1 / 2}}{\Gamma(n / 2)}(n+1+2 k)^{(n-1) / 2} \\
& \cdot\left[\frac{\Gamma(k+1+n / 2)}{2 \sqrt{\pi} \Gamma(k+1+(n+1) / 2)}\right]^{(n-1) /(n+1+2 k)}, \\
C_{2}(n, k)= & \left(\frac{1}{n-1}\right)^{1+(n-3-2 k) /(n+1+2 k)} \cdot 2^{(n+1) / 2} \pi^{n / 2} \frac{[n !]^{1 / 2}}{\Gamma(n / 2)} \cdot\left(\frac{n+1}{2}+k\right)^{(n-1) / 2} \\
& \cdot \frac{\Gamma(k+1+n / 2)}{\Gamma(k+2) \Gamma(n / 2)} \cdot\left[\frac{2 \sqrt{\pi} \Gamma(k+1+(n+1) / 2)}{\Gamma(k+1+n / 2)}\right]^{(2 k+3-n) /(n+1+2 k)} \\
C_{3}(n, k)= & \frac{2 \pi^{n / 2}}{\Gamma(n / 2)}[n !]^{1 / 2}[n+1+2 k]^{(n-1) / 2} \\
& \cdot\left[\frac{\Gamma(k+1+n / 2)}{2 \sqrt{\pi} \Gamma(k+1+(n+1) / 2)}\right]^{(n-1) /(n+1+2 k)}, \\
C_{4}(n, k)= & {[\sqrt{\pi}(n+1)(n+1+2 k)]^{(k+1) /((n+1) / 2+k)} \cdot \frac{\Gamma(k+1+n / 2)}{\Gamma(k+2) \Gamma(n / 2)} . }
\end{aligned}
$$

* For $m \gg n$ we can replace $(\ln m) m$ by

$$
(\ln m)^{(n+1+4 k+2) /(n+1+2 k)} m^{(n+1-2) /(n+1+2 k)} \cdot C_{4}(n, k) .
$$


Apparently the $m$-dependency is dominated by the result from $\sum_{B S \in \mathfrak{F}_{1}} s\left(h_{B S}\right)$ of

$$
(m+1)^{1+(n-3-2 k) /(n+1+2 k)}=(m+1)^{2((n-1) /(n+1+2 k))}=(m+1)^{2-(4+4 k) /(n+1+2 k)} .
$$

This holds as long as $k<(n-3) / 2$. After that the sorting term and heap effort become the strongest.

Theorem 7. For our special cases we obtain the following dependency on $m(m \rightarrow \infty$, $n$ fixed):

$$
\begin{aligned}
& \left.k \rightarrow-1 \quad \text { (uniform distribution on } \omega_{n}\right) \quad m^{2((n-1) /(n+1+2 k))} \underset{k \rightarrow-1}{\longrightarrow} m^{2} . \\
& k=0 \quad \text { (uniform distribution on } \Omega_{n} \text { ) } \quad m^{2((n-1) /(n+1))}=m^{2-4 /(n+1)} \text {. } \\
& k>\frac{n-3}{2} \text { (distribution centrally directed) } m \text {. } \\
& k=\frac{n-1}{2} \quad \text { (symmetric radial density) } \quad m \text {. } \\
& \text { (4) } k \rightarrow \infty \text { (totally centralized) } m \text {. }
\end{aligned}
$$

Remark. The question remains whether the Throw-Away Principle could also be combined with Gift-Wrapping without storage. However, this seems to be inefficient in most cases. Remember that Avis and Fukuda [2] work with a worst-case bound of $O(m \cdot n \cdot \# \mathfrak{F})$. If we want to implement the Throw-Away idea, we must calculate, for each point check, the potential height of the preliminary facet, but Avis and Fukuda make the evaluation of all rows (instead of one row) necessary, because every row is a candidate for a reverse pivot step and no other information (storage) is available. So we would have to accept another factor $n$ in the main term of our result, which means

$$
\begin{array}{ll}
E\left(n^{2} \sum_{B S \in \mathfrak{F}} s\left(h_{B S}\right)\right) & \text { for the combination instead of } \\
E\left(n \sum_{B S \in \mathfrak{F}} s\left(h_{B S}\right)\right) & \text { for pure Throw-Away Principle. }
\end{array}
$$

This drawback has to be weighed against the advantage of saving the storage effort of $(\# \mathfrak{F}) \cdot n^{2} \ln m$.

So, in almost all cases the combination is inferior to the pure Throw-Away Principle.

Remark. The analysis finished above considers rotation-symmetric distributions about the origin, and all point distances are measured with respect to the origin. In practical situations, it would be preferable to make a transformation of coordinates. Choose $\bar{a}$ (the barycenter of $\left.\mathrm{CH}\left(a_{1}, \ldots, a_{m}\right)\right)$ as the new origin. This has two advantages:

- The collection of points is somehow "rotation-symmetric" about the barycenter (often even when the points do not come from a rotation-symmetric distribution).

- There are no facets of the second kind. This fact simplifies the algorithm and lets us fully profit from the saving effect analyzed in this paper. 
In contrast to those advantages, there is one theoretical drawback: Since the barycenter $\bar{a}$ depends on $\left\{a_{1}, \ldots, a_{m}\right\}$, our new points $a_{1}-\bar{a}, \ldots, a_{m}-\bar{a}$ are no longer independent random variables. This would dramatically complicate any attempt for a probabilistic analysis.

However, in the asymptotic case $m \rightarrow \infty, n$ fixed, the dependence gets extremely small. In that case we can trust our calculated results even if we do the practice-oriented barycenter transformation.

\section{References}

1. Akl, S. G., and Toussaint, G. T. A fast convex hull algorithm. Information Processing Letters 7 (1978), 219-222.

2. Avis, D., and Fukuda, K. A pivoting algorithm for convex hulls and vertex enumeration of arrangements and polyhedra. Discrete \& Computational Geometry 8 (1992), 295-313.

3. Bentley, J., and Shamos, M. Divide and conquer for linear expected time. Information Processing Letters 7(2) (1978), 87-91.

4. Borgwardt, K. H. The Simplex method. A probabilistic analysis. In Algorithms and Combinatorics, Vol. 1, Graham, R. L., Korte, B., and Lovász, L., eds., Springer-Verlag, Berlin, 1987.

5. Borgwardt, K. H., Gaffke, N., Jünger, M., and Reinelt, G. Computing the convex hull in the Euclidean plane in linear expected time. In Applied Geometry and Discrete Mathematics (The Victor Klee Festschrift), Vol. 4, Gritzmann, P., and Sturmfels, B., eds., DIMACS Series in Discrete Mathematics and Theoretical Computer Science, 1991, pp. 91-107.

6. Buchta, C. Zufällige Polyeder-eine Übersicht. In Zahlentheoretische Analysis, Lecture Notes in Mathematics, Vol. 1114, Hlawka, E., ed., Springer-Verlag, Berlin, 1985, pp. 1-13.

7. Chand, D. R., and Kapur, S. S. An algorithm for convex polytopes. Journal of the Association for Computing Machinery 17(1) (1970), 78-86.

8. Clarkson, K. L. Linear programming in $O\left(n 3^{d \times d}\right)$ time. Information Processing Letters 22(1) (1986), 21-24.

9. Clarkson, K. L., and Shor, P. W. Applications of random sampling in computational geometry, II. Discrete \& Computational Geometry 4 (1989), 387-421.

10. Devroye, L. P. How to reduce the average complexity of convex hull finding algorithms. Computers and Mathematics with Applications 7 (1981), 299-308.

11. Devroye, L. P. Moment inequalities for random variables in computational geometry. Computing 30 (1983), 111-119.

12. Devroye, L. P., and Toussaint, G. T. A note on linear expected time algorithms for finding convex bulls. Computing 26 (1981), 361-366.

13. Dwyer, R. A. Average-Case Analysis of Algorithms for Convex Hulls and Voronoi Diagrams. CMU-CS88-132, Computer Science Dept., Carnegie-Mellon University, 1988.

14. Dwyer, R. A. On the convex hull of random points in a polytope. Journal of Applied Probability 25 (1988), 688-699.

15. Dwyer, R. A. Random convex hulls in a product of balls. Probability Theory and Related Fields 86 (1990), 457-467.

16. Dwyer, R. A. Convex hulls of samples from spherically symmetric distributions. Discrete Applied Mathematics 31 (1991), 113-132.

17. Dyer, M. E. Linear time algorithms for two- and three-variable linear programs. SIAM Journal of Computing 13(1) (1984), 31-45.

18. Dyer, M. E., and Frieze, A. M. A randomized algorithm for fixed-dimensional linear programming. Mathematical Programming 44 (1989), 203-212.

19. Graham, R. L. An efficient algorithm for determining the convex hull of a finite planar set. Information Processing Letters 1 (1972), 132-133.

20. Jünger, M., and Reinelt, G. Fast Computation of Convex Hulls. Schwerpunktprogramm der DFG: Anwendungsbezogene Optimierung und Steuerung, No. 137, Universität Augsburg, 1989. 
21. Kallay, M. Convex Hull Algorithms in Higher Dimensions. Dept. Mathematics, University of Oklahoma, 1981.

22. Küfer, K. Asymptotische Varianzanalysen in der stochastischen Polyedertheorie. Doktorarbeit, Universität Kaiserslautern, 1992.

23. Megiddo, N. Linear programming in linear time when the dimension is fixed. Journal of the Association for Computing Machinery 31 (1984), 114-127.

24. Seidel, R. A Convex Hull Algorithm Optimal for Points in Even Dimensions. Master's thesis, University of British Columbia, 1981.

25. Seidel, R. Constructing higher dimensional convex hulls at logarithmic cost per face. Proceedings of the 18th Annual SIGACT Symposium, 1986, pp. 404-413.

26. Seidel, R. Small-dimensional linear programming and convex hulls made easy. Discrete \& Computational Geometry 6 (1991), 423-434.

27. Swart, G. Finding the convex hull facet by facet. Journal of Algorithms 6 (1985), 17-48.

28. Van Wel, B. F. The convex hull of a uniform sample from the interior of a simple $d$-polytope. Journal of Applied Probability 26 (1989), 259-273.

29. Wendel, J. G. A problem in geometric probability. Mathematica Scandinavica 11 (1962), 109-111.

Received August 29, 1994, and in revised form May 15, 1996. 